\title{
Oncolytic virotherapy for small-cell lung cancer induces immune infiltration and prolongs survival
}

\author{
Patrick Kellish, ${ }^{1}$ Daniil Shabashvili, ${ }^{1}$ Masmudur M. Rahman, ${ }^{2}$ Akbar Nawab, ${ }^{1}$ Maria V. Guijarro, ${ }^{1}$ Min Zhang, ${ }^{3}$ Chunxia Cao, ${ }^{3}$ \\ Nissin Moussatche, ${ }^{2}$ Theresa Boyle, ${ }^{4}$ Scott Antonia, ${ }^{4}$ Mary Reinhard, ${ }^{5}$ Connor Hartzell, ${ }^{1}$ Michael Jantz, ${ }^{3}$ Hiren J. Mehta, ${ }^{3}$ \\ Grant McFadden, ${ }^{2}$ Frederic J. Kaye, ${ }^{3}$ and Maria Zajac-Kaye ${ }^{1}$
}

'Department of Anatomy and Cell Biology, ${ }^{2}$ Department of Molecular Cenetics and Microbiology, ${ }^{3}$ Department of Medicine, University of Florida, Gainesville, Florida, USA. ${ }^{4}$ Moffitt Cancer Center, Tampa, Florida, USA. ${ }^{5}$ Department of Veterinary Pathology, University of Florida, Gainesville, Florida, USA.

\begin{abstract}
Oncolytic virotherapy has been proposed as an ablative and immunostimulatory treatment strategy for solid tumors that are resistant to immunotherapy alone; however, there is a need to optimize host immune activation using preclinical immunocompetent models in previously untested common adult tumors. We studied a modified oncolytic myxoma virus (MYXV) that shows high efficiency for tumor-specific cytotoxicity in small-cell lung cancer (SCLC), a neuroendocrine carcinoma with high mortality and modest response rates to immune checkpoint inhibitors. Using an immunocompetent SCLC mouse model, we demonstrated the safety of intrapulmonary MYXV delivery with efficient tumor-specific viral replication and cytotoxicity associated with induction of immune cell infiltration. We observed increased SCLC survival following intrapulmonary MYXV that was enhanced by combined low-dose cisplatin. We also tested intratumoral MYXV delivery and observed immune cell infiltration associated with tumor necrosis and growth inhibition in syngeneic murine allograft tumors. Freshly collected primary human SCLC tumor cells were permissive to MYXV and intratumoral delivery into patient-derived xenografts resulted in extensive tumor necrosis. We confirmed MYXV cytotoxicity in classic and variant SCLC subtypes as well as cisplatin-resistant cells. Data from 26 SCLC human patients showed negligible immune cell infiltration, supporting testing MYXV as an ablative and immune-enhancing therapy.
\end{abstract}

\section{Introduction}

Small-cell lung cancer (SCLC) is an aggressive neuroendocrine subtype that was recently selected by the National Cancer Institute, $\mathrm{NIH}$, for a focused strategic plan due to its high incidence in the United States (35,000 new cases in 2017), the lack of improvement on 5-year survival rates, and as a direct response to the Recalcitrant Cancer Research Act H.R.733 passed by Congress (1, 2). Despite uncertainty in the cell subtype of origin (3), SCLC has a unique genetic and clinical phenotype with concurrent mutational inactivation of the RB1 and p53 genes $(4,5)$ associated with invasive tumor growth and early metastatic dissemination. Although SCLC is initially sensitive to standard cytotoxic chemotherapy and radiation therapy, tumor responses are short-lived (2). Despite recent studies suggesting a role for immune checkpoint inhibition, clinical investigational trials over the past 4 decades have been unable to substantially impact the cure rate of patients who present with advanced disease (6-8). Early studies noted markedly reduced expression of HLA-A, -B, -C, and beta2-microglobulin genes in SCLC tumor lines and primary samples, which could

Authorship note: PK and DS contributed equally to this work.

Conflict of interest: GM is cofounder of Oncomyx, which is developing myxoma virus as an oncolytic therapeutic.

Copyright: @ 2019, American Society for Clinical Investigation

Submitted: May 18, 2018; Accepted: March 14, 2019; Published: April 29, 2019.

Reference information: J Clin Invest. 2019;129(6):2279-2292.

https://doi.org/10.1172/JCl121323. impact antitumor immunity (9-11). Recent studies show PD-L1 biomarker staining is low in most SCLC samples compared with non-SCLC samples (12-14). Additionally, a longitudinal study of lung adenocarcinoma that transformed into SCLC morphology was also associated with loss of PD-L1 expression and reduced numbers of tumor-infiltrating lymphocytes (15). Since induction of host immune cell infiltration is a parameter associated with durable antitumor efficacy of immune checkpoint inhibition (16-18), we were interested in testing the ability of a modified oncolytic virus to enhance tumor response and immune cell infiltration using immunocompetent genetically engineered mouse SCLC and allograft models.

Oncolytic viruses are replication-competent viruses that selectively infect and kill tumor cells in vitro and in vivo while sparing normal cells and tissues $(19,20)$. These observations led to testing oncolytic virotherapy for treatment of different cancer subtypes where standard treatments had otherwise failed. Results from early clinical trials were mixed $(21,22)$, and subsequent protocols focused on enhancing efficiency of tumor-specific viral replication associated with direct tumor cell cytotoxicity. In addition, there is growing recognition that oncolytic viruses have the potential to generate new antitumor host immune responses (23). For example, a conditionally replicative modified herpes viral vector expressing GM-CSF was the first oncolytic virus approved by the FDA, after demonstrating efficacy in a phase III clinical study in patients with advanced unresectable melanoma $(24,25)$. Further, recent data using this vector (talimogene laherparepvec) 
suggested an important role for oncolytic virotherapy in modulating host immune responses where tumor response in patients with advanced melanoma was directly correlated with induction of $\mathrm{T}$ cell infiltration and reexpression of PD-L1 and other IFN- $\gamma$ biomarkers $(16,26)$.

Myxoma virus (MYXV) is a distinct Leporipoxvirus initially identified as the causative agent for myxomatosis, a lethal disease specific to European rabbit strains (oryctolagus cuniculus) but nonpathogenic for other mammals $(27,28)$. Based on this observation, MYXV was released in Australia in the 1950s to control feral rabbits (29). This is credited as the first biological control program of a mammalian pest that also confirmed lack of toxicity to other mammalian species, including humans. Although a rabbit-specific poxvirus, MYXV is able to infect and replicate in tumor cells derived from diverse species, and oncolytic activity has been demonstrated for several human tumor lineages $(30,31)$. There are no unique tumor-specific surface receptors for MYXV, and modulation by host DEAD-box helicases (32) combined with the inability of tumor cells to induce protective antiviral host cytokine responses are proposed as reasons underlying specificity for MYXV replication and cytotoxicity in human tumor cells but not normal tissues $(32,33)$. We selected human SCLC as a solid tumor model for preclinical testing for MYXV therapy because (a) relapsed SCLC is incurable (8), (b) RB and p53 are targeted for mutational inactivation in essentially $100 \%$ of SCLC (34) and PTEN loss and/or AKT1 activation are concurrently observed in a large subset of tumor samples $(35,36)$, and this somatic mutational pattern is specifically associated with enhanced efficiency for MYXV infection and replication in tumor cells $(37,38)$, and (c) the recent demonstration of tumor response rates to immune checkpoint inhibition $(13,39)$ offered an opportunity to explore combined immunotherapy with ablative oncolytic treatments to increase the immunogenic profile of target tumor tissues and improve the outcome of these patients with advanced disease.

\section{Results}

Efficient MYXV viral replication in human SCLC floating spheroid cell clusters and variant adherent cell lines. To study efficiency of MYXV virotherapy against human SCLC cell lines in vitro, we used vMyx-GFP-TdT (GFP under control of a poxvirus early/late promoter and TdTomato under poxvirus p11 late promoter) to score for early and late viral replication phases, respectively. We observed GFP expression, indicating infection/early gene expression, and TdTomato expression, indicating viral replication in both adherent and nonadherent human SCLC cell lines (Figure $1, \mathrm{~A}$ and $\mathrm{B})$. In addition, we performed flow cytometry on normal lung epithelial cells and 3 human SCLC cell lines and observed efficient viral infection for each human SCLC cell line tested, with negligible effect on normal cells (Figure 1C; additional quantitation in Supplemental Figure 1, A and B). We also measured viral replication efficiency in 2 human SCLC lines at 24 and 48 hours after initial (1 hour) viral attachment and detected at least a 1 log increase in viral titers (Figure 1D). In addition, we tested a modified vMyx-M135KO-GFP (viral M135 gene replaced with GFP, ref. 40), which exhibits attenuated disease in European rabbits but retains enhanced oncolytic properties in cancer cells (41), and therefore proposed it as an optimal agent for clinical phase I test- ing (Supplemental Figure 2). Since vMyx-M135KO-GFP lacks the $\mathrm{TdT}$ reporter, we performed electron microscopy to confirm viral replication with detection of mature and immature viral progeny formation in human SCLC cells (Figure 1E). To determine if MYXV infection elicits an immunogenic cell death, we performed an ATP release assay following MYXV infection (42). We observed a rapid increase in ATP release 4 hours after exposure to either MYXV or the modified vMyx-M135KO-GFP compared with uninfected control cells (Figure $1 \mathrm{~F}$ ), consistent with an immunogenic cell death mechanism for SCLC cells following MYXV exposure. Based on these results, both parental MYXV and modified vMyxM135KO-GFP were utilized in subsequent in vivo mouse studies.

While SCLC cell lines usually grow as floating spheroid clusters (classic nonadherent phenotype), a subset exhibit an adherent monolayer (variant) morphology $(43,44)$. Since Seneca Valley picornavirus was previously shown to preferentially infect variant SCLC cells (45), we tested both floating spheroid (cell lines H60, H69, H82) and adherent monolayer (cell lines H372, H446, H1048) subtypes. We observed efficient infection and late viral replication in all tested human SCLC cell lines independent of cell morphology phenotype for both MYXV and the modified vMyxM135KO-GFP backbone (Figure 1, Supplemental Figure 2).

SCLC patient biopsy specimens and an optimized genetically engineered murine SCLC model show scant host immune cell infiltration, and murine SCLC cell lines show efficient MYXV infection and replication. Prior studies have noted that the aggressive phenotype of human SCLC is associated with reduced expression of immune markers and lower PD-L1 biomarker staining compared with nonSCLC samples (9-14). We performed immunohistochemistry on a set of 26 new human SCLC tumor biopsies and observed that 18 of 26 samples (70\%) were negative (0 score) for presence of infiltrating CD $45^{+}$immune cells and only 1 of 26 samples (4\%) exhibited a score of 3 or higher (Figure 2A). All samples were scored by the same pathologist using previously established cut-off criteria, as described in Methods. Tabulated data are included in Supplemental Figure 3.

To test the efficiency of MYXV virotherapy as an effective immunoablative agent in vivo using an immunocompetent SCLC genetically engineered mouse model (GEMM), we first optimized the $\mathrm{p} 53^{-/-} / \mathrm{Rb}^{-/-} / \mathrm{p} 130^{-/-}$conditional mouse model (46) by limiting dilution of intratracheal adenovirus Cre-recombinase (Ad-Cre) and determined $1 \times 10^{6}$ focus-forming units (FFU) was sufficient to consistently generate fewer SCLC tumorlets per lung section, which more closely simulates human disease (Figure 2B). Undifferentiated neuroendocrine tumorlet foci arising after intratracheal Ad-Cre delivery were confirmed by positive NCAM-1 (CD56) staining (Figure 2C). To examine end-stage advanced disease, we obtained lungs from mice 8-9 months after Ad-Cre delivery and observed SCLC tumors occupying large regions of the lung (Figure 2D). These end-stage SCLC tumors showed scant $\mathrm{CD} 45^{+}$and $\mathrm{CD}^{+}$immune cell infiltration as determined by IHC (Figure 2D). Representative flow cytometry analysis of whole-lung, single-cell suspensions confirmed a marked reduction in $\mathrm{CD}_{4} 5^{+}$and $\mathrm{CD}^{+}$immune cell populations in lungs from advanced SCLC compared with a normal adult mouse lung control (Figure 2E). Quantification was performed in 3 independent mice and showed a statistically significant reduc- 
A
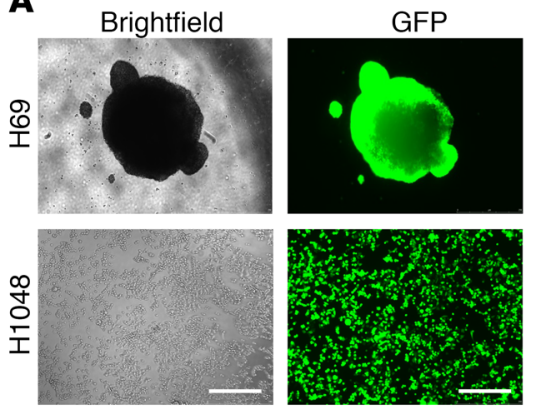

C
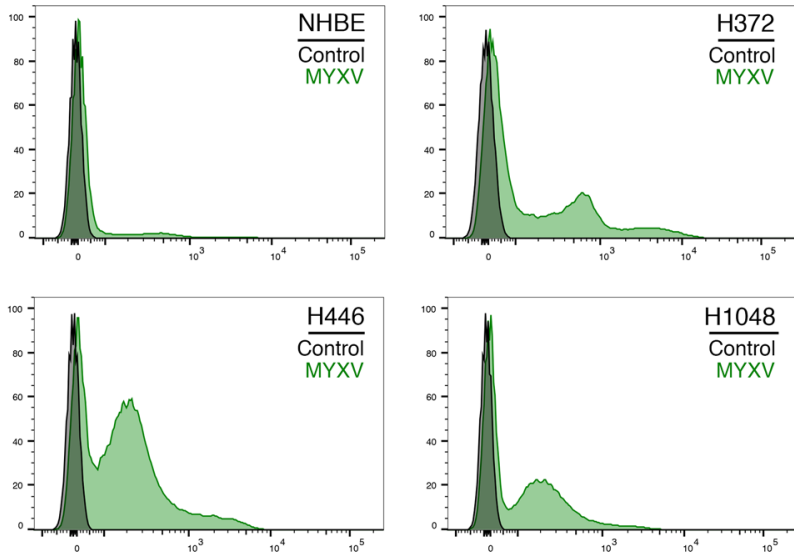

GFP (infection)

E

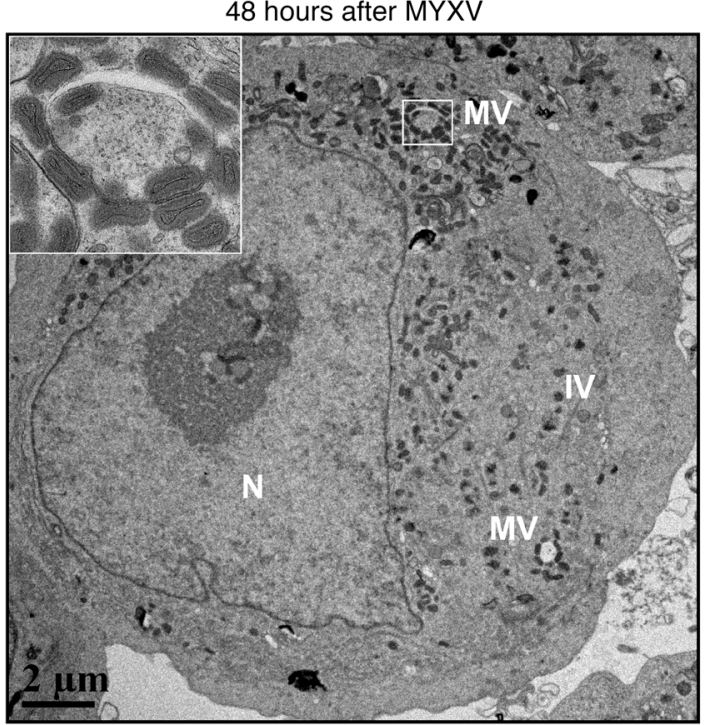

$\mathbf{F}$
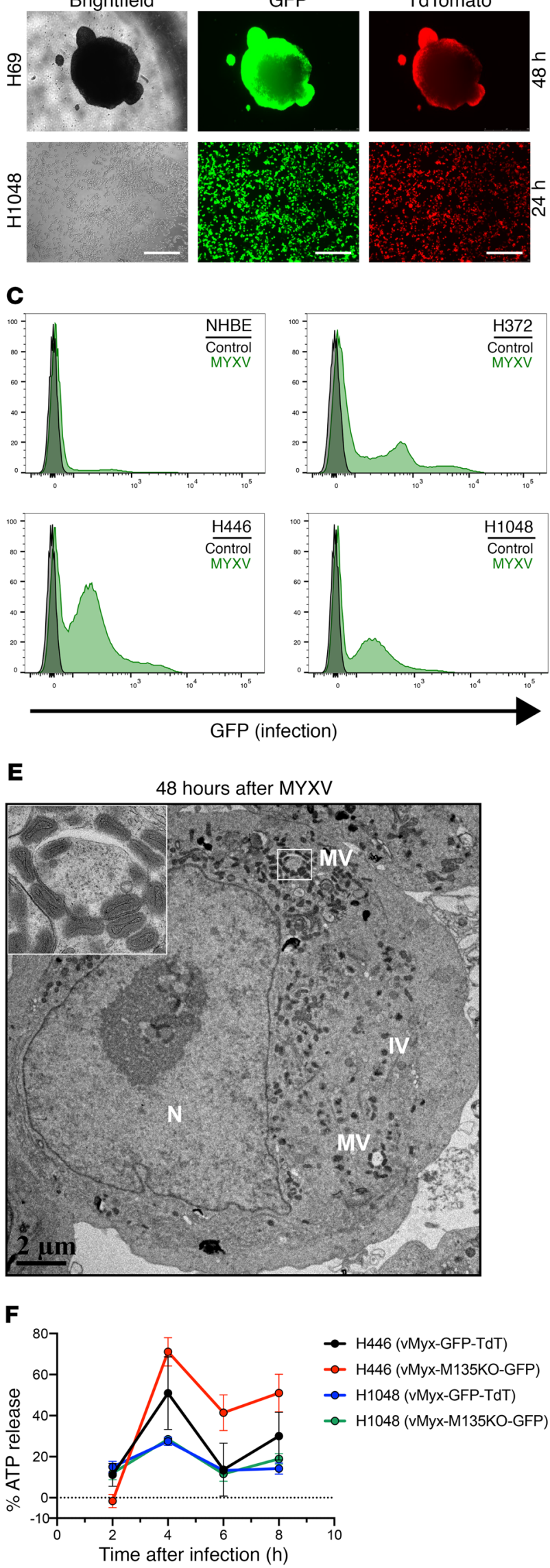

B
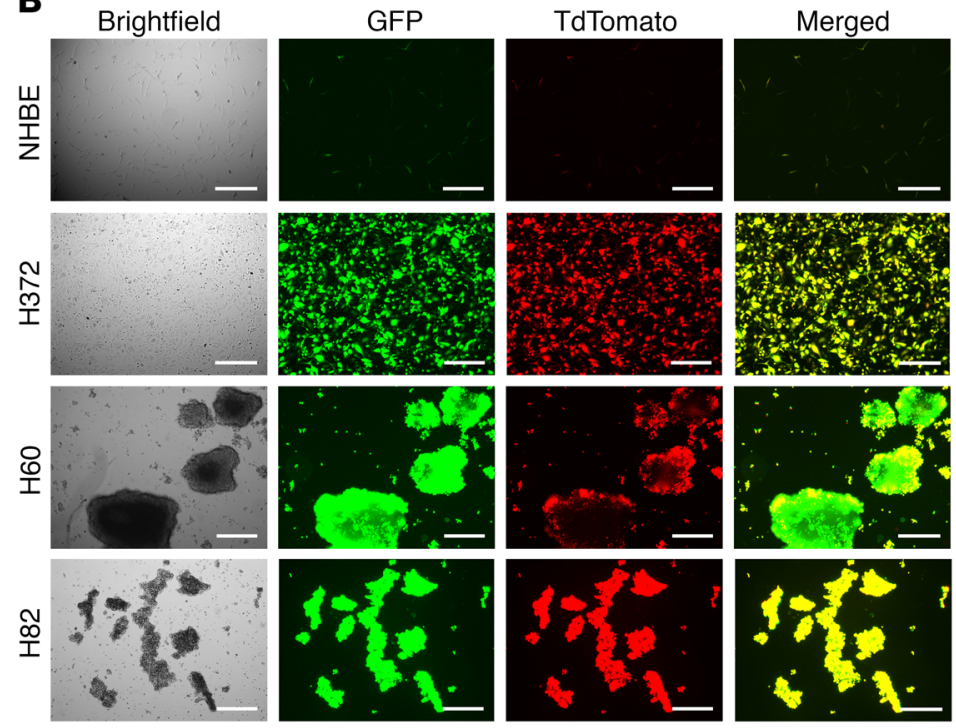

D

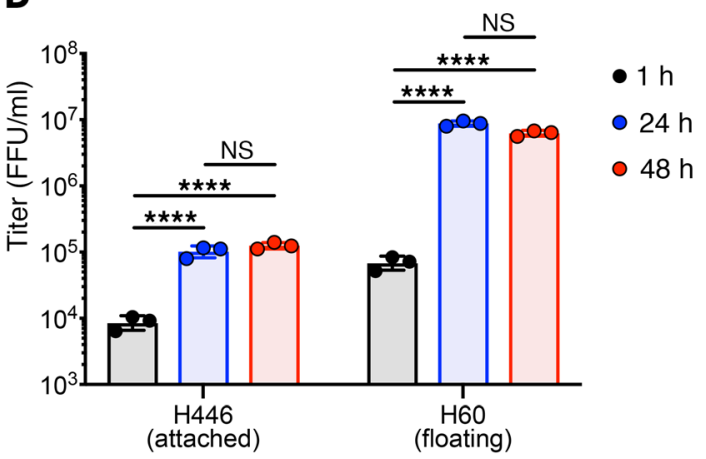

Figure 1. MYXV infects and replicates in human SCLC cells. (A) Human SCLC cell lines infected with vMyx-GFP-TdT at $10 \mathrm{MOI}$ demonstrating both early (GFP) and late (TdTomato) gene expression for viral replication at 48 hours for cell line $\mathrm{H} 69$ and 24 hours for cell line H1048. Scale bars, $200 \mu \mathrm{m}$. (B) Expanded human SCLC and control NHBE cell line infected with vMyx-CFP-TdT at $10 \mathrm{MOI}$. All SCLC cell lines demonstrate both early (GFP) and late (TdTomato) gene expression for replication at 48 hours whereas control cell lines show negligible permissiveness. Scale bars, $50 \mu \mathrm{m}$. (C) Quantification of early gene expression 18 hours after infection ( $1 \mathrm{MOI}$ ). For each cell line, the flow cytometry histograms compare vMyx-GFP-TdT-treated cells relative to control cells (no infection).

(D) Replication of MYXV in human SCLC cell lines illustrated by the formation of viral progeny 24 and 48 hours after vMyx-CFP-TdT infection. Results are representative of 3 replicates per group, bar showing mean \pm SD with all data points. ${ }^{* * *} P<0.0001$ by 1 -way ANOVA and Tukey's multiple comparison test. (E) Transmission electron microscopy of human SCLC H6O cell line 48 hours after infection with vMyx-M135KO-GFP at $10 \mathrm{MOI}$. Mature virus (MV), immature particles (IV), and nucleus (N) are labeled. MV is visualized in several areas of the cytoplasm. Inset highlights the indicated region showing MV. Scale bar, $2 \mu \mathrm{m}$. (F) Immunogenic cell death (ICD) assay illustrates the increase in ATP release compared with control cells (no infection). Results are representative of 4 replicates per group and time point. Data represent mean \pm SEM. 
A

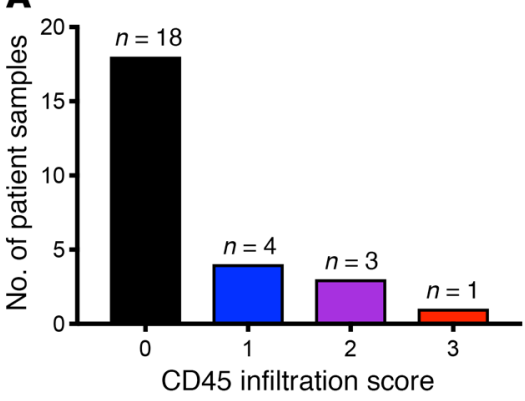

D

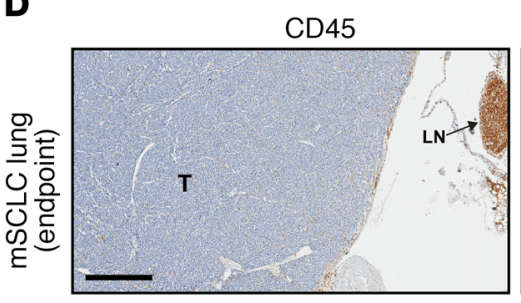

F
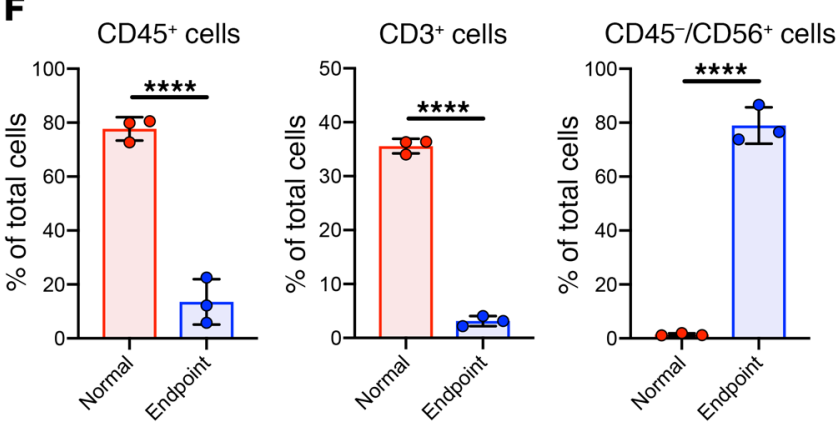

B

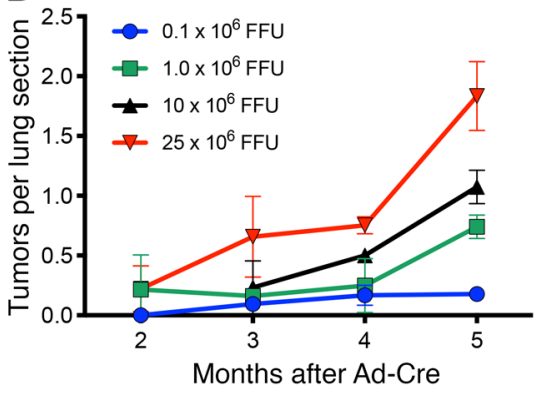

$\mathbf{C}$
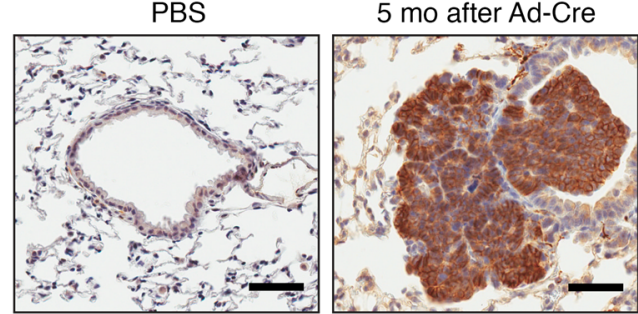

E

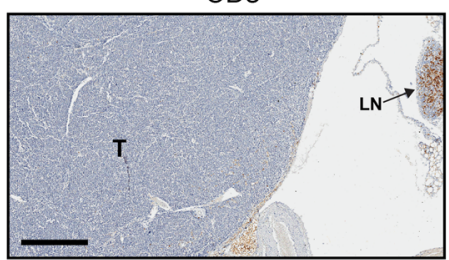

CD3
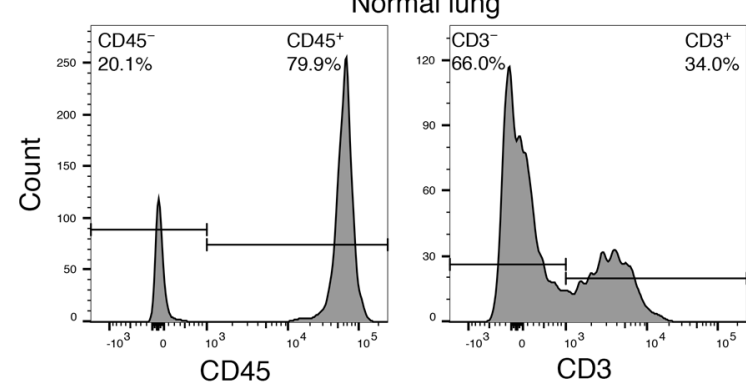

CD3

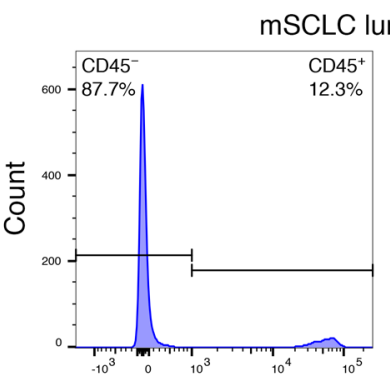

CD45

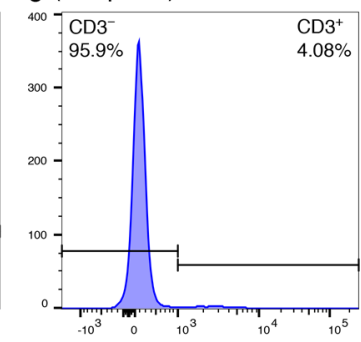

CD3

Figure 2. SCLC patient specimens and a genetically engineered mouse model for SCLC show scant immune cell infiltration. (A) Data from 26 SCLC patient specimens showing CD45 infiltration score $(0-3+)$ defined by $\mathrm{CD} 45^{+}$immunostaining. (B) Dose-dependent study of Ad-Cre delivered by intratracheal injection showing the number of tumor lesions observed at the indicated time points for each Ad-Cre dose. Results are representative of 3 animals per group, and data indicate mean \pm SD. (C) NCAM-1 (CD56) immunohistochemistry 5 months after intratracheal delivery of Ad-Cre compared with PBS-treated

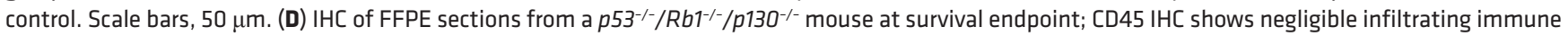
cells and CD3 IHC shows negligible infiltrating T lymphocytes. The advanced SCLC lesion (T) occupies majority of the section with lymph node (LN) upper right confirming CD45 and CD3 reactivity. Scale bars, $400 \mu \mathrm{m}$. (E) CD45 and CD3 populations from an enzymatically digested whole lung obtained from adult

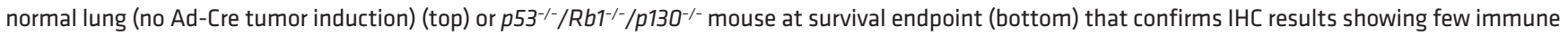
cells (CD45 $)$ and T lymphocytes (CD3+) in endpoint SCLC lung. (F) Quantification from whole-lung tissue using control (no Ad-Cre induction; $n=3$ ) and SCLC endpoint $(n=3)$ showing reduction in immune $\mathrm{CD}_{4} 5^{+}$or $\mathrm{CD} 3^{+}$cells and presence of CD45-/CD56 $\mathrm{SCLC}$ tumor cells. Bar showing mean $\pm \mathrm{SD}$ with all data points. ${ }^{* * *} P<0.0001$ by unpaired Student's $t$ test.

tion in immune cell infiltration in endpoint lungs carrying a high CD45 /CD56 ${ }^{+}$SCLC tumor burden compared with lungs from control mice (Figure 2F). These data suggest tumorlets advancing to end-stage SCLC are associated with reduced potential for cancer immune surveillance.

We also dissected individual SCLC foci to generate syngeneic murine SCLC cell lines that exhibited either adherent (A) or floating spheroid (F) phenotypes. We observed efficient MYXV infection, with early (GFP) and late (TdTomato) gene expression (Figure 3A). Quantification of MYXV infection and early gene expression was performed by flow cytometry and demonstrates efficient infection observed in murine SCLC with a negligible effect on normal murine embryonic fibroblast (MEF) cells (Figure 3B, Supplemental Figure 1). As tested earlier for human SCLC cells, we quantitated the generation of infectious MYXV progeny in murine SCLC tumor lines and observed a statistically significant enhancement in viral replication (Figure 3C, Supplemental Figure 4). In addition, as observed earlier with human SCLC cell lines, we detected similar infection efficiency using the modified vMyx-M135KO-GFP in murine SCLC cell lines (Supplemental Figure 5). Five of the 6 human SCLC cell lines tested were generated from patients who had previously received combination chemotherapy, whereas the murine SCLC cell lines were chemotherapy-treatment naive. Since a phase I clinical trial testing oncolytic virotherapy would enroll relapsed SCLC patients associated with the phenotype of cisplatin resistance, we exposed murine SCLC cell line 2.1A to continuous cisplatin with periodic high concentration cisplatin pulses to generate the cisplatin- 
A
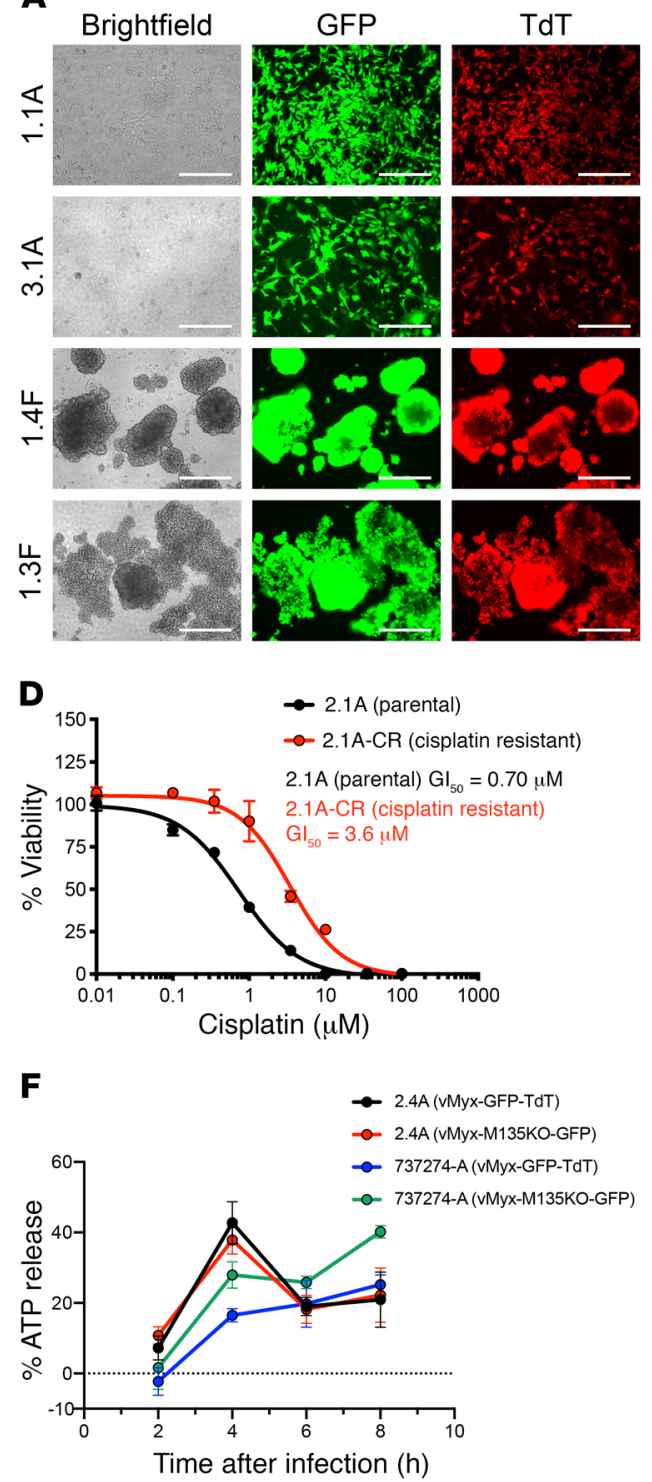

B

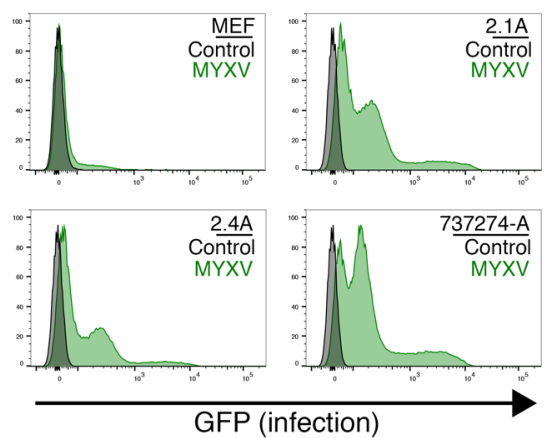

E
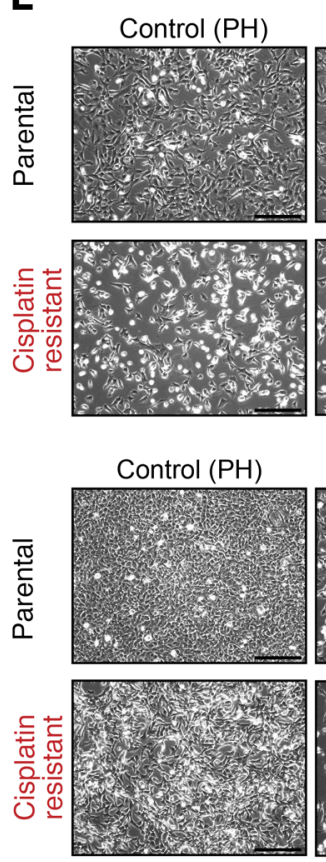

MYXV (PH)
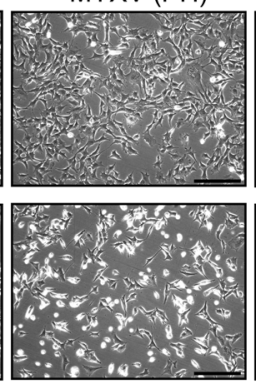

MYXV (PH)
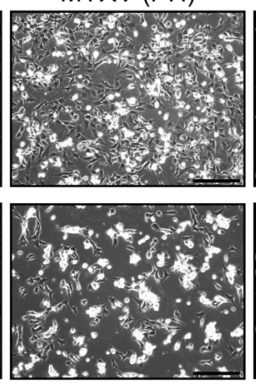

C

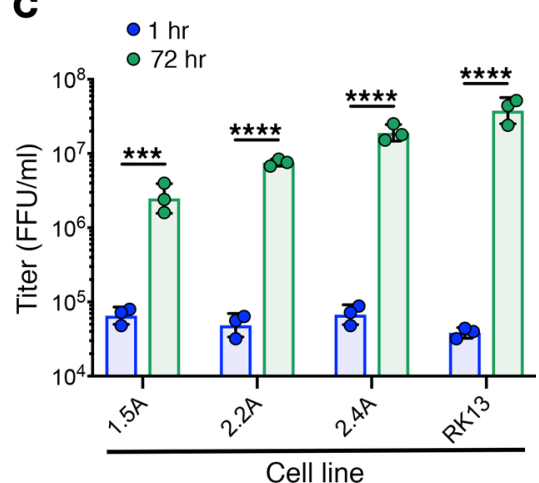

MYXV (GFP)
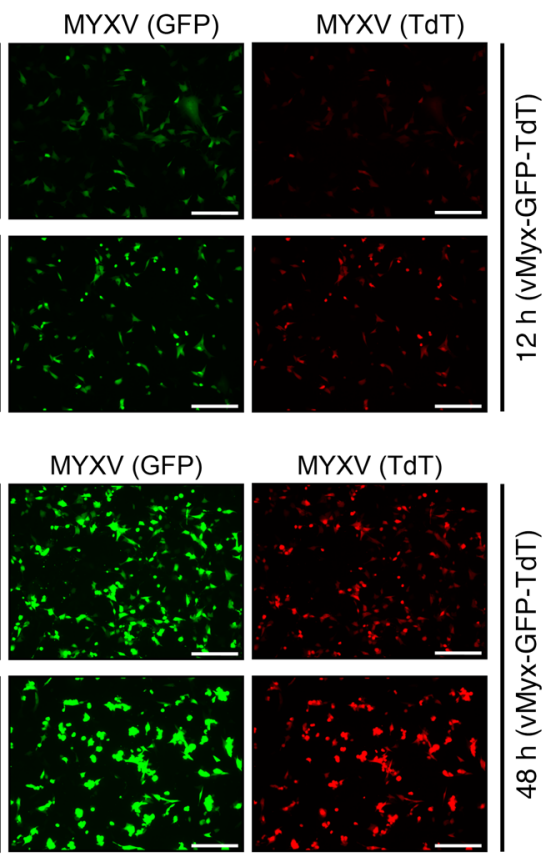

MYXV (TdT)

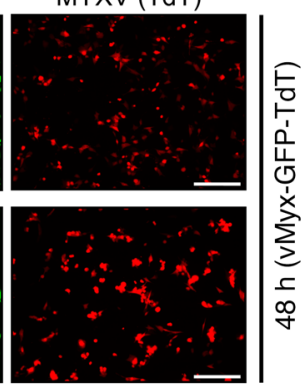

Figure 3. MYXV efficiently infects and replicates in murine SCLC and cisplatin-resistant cell lines derived from an optimized GEMM. (A) Murine SCLC cell lines infected with vMyx-GFP-TdT at $10 \mathrm{MOI}$ demonstrating both early (GFP) and late (TdTomato) gene expression for replication at 48 hours. Scale bars, $250 \mu \mathrm{m}$. (B) Quantification of early gene expression 18 hours after infection (1 MOI) for each cell line. The flow cytometry histograms compare vMyx-GFPTdT-treated cells relative to control cells (no infection). (C) Replication of MYXV in murine SCLC cells and a permissive RK13 control cell line illustrated by the formation of viral progeny 72 hours after vMyx-GFP-TdT infection compared with 1 hour (input viral attachment stage). Results are representative of 3 replicates per group. Bar shows mean \pm SD with all data points. ${ }^{* *} P<0.001,{ }^{* * * *} P<0.0001$, by unpaired Student's $t$ test. (D) Viability assay showing a greater than 5 -fold increase in $\mathrm{GI}_{50}$ for the cisplatin-resistant murine SCLC cell line compared with the matched parental murine SCLC cell line. Data represent mean \pm SD for triplicate samples. (E) Cisplatin-resistant murine SCLC cell lines and the matched parental murine SCLC cell line infected with vMyx-GFP-TdT at $10 \mathrm{MOI}$ demonstrating both early (GFP) and late (TdTomato) gene expression at 12 and 48 hours. Scale bars, $250 \mu \mathrm{m}$. (F) Immunogenic cell death (ICD) assay illustrated the increased ATP release compared with control cells (no infection). Results are representative of 4 replicates per group and time point. Data represent mean \pm SEM. A, adherent phenotype; F, floating spheroid phenotype.

resistant cell line 2.1A-CR with greater than 5 -fold cisplatin resistance (Figure 3D). We show efficient MYXV infection and late viral gene expression/replication in both the cisplatin-resistant cell line $(2.1 \mathrm{~A}-\mathrm{CR})$ and its matched parental treatment-naive cell line (2.1A) (Figure 3E). To determine if murine SCLC undergoes immunogenic cell death following MYXV infection, we performed an ATP release assay. Similar to data collected from human SCLC, we observed a rapid increase in ATP release at 4 hours when compared with ATP release in uninfected control cells, indicating immunogenic cell death following MYXV exposure in murine SCLC (Figure 3F).

Intrapulmonary MYXV delivery to immunocompetent SCLC GEMM mice induces prolonged host immune cell infiltration in tumor-bearing mice. To test intrapulmonary MYXV delivery, we performed intranasal instillation using $5 \times 10^{7} \mathrm{FFU}$ vMyx-FLuc (Firefly luciferase under control of a poxvirus early/late promoter) 3 months after intratracheal Ad-Cre delivery. We observed luciferase signals in bilateral lungs at 3 days $(n=3$ mice) with intrapul- 
A

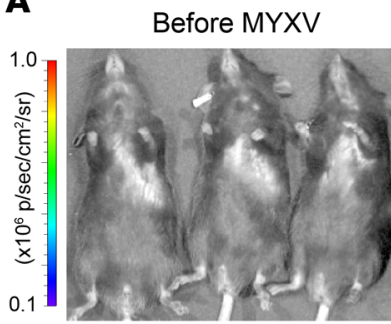

3 days after MYXV

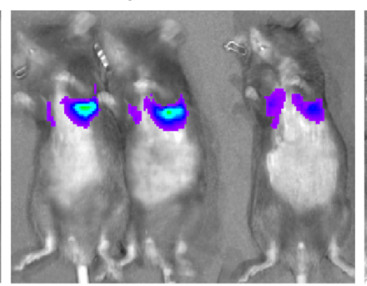

B

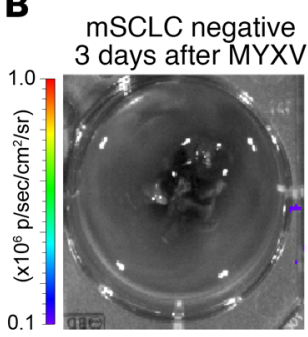

mSCLC positive 3 days after MYXV

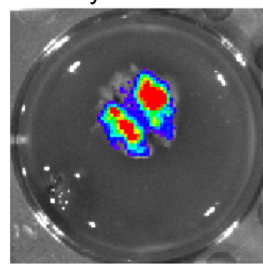

mSCLC positive

7 days after MYXV

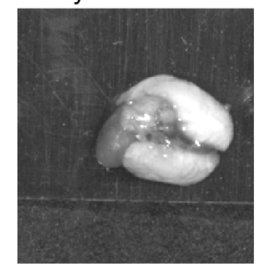

C

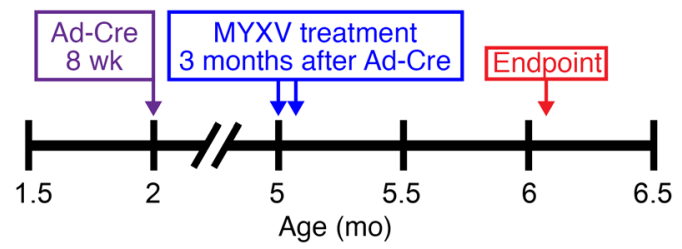

D

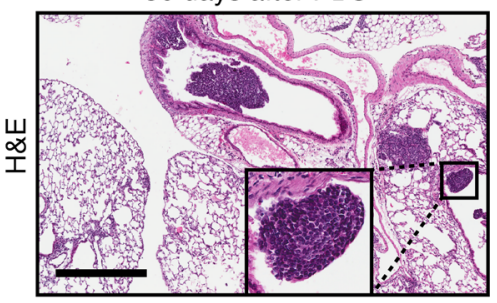

$\mathbf{E}$

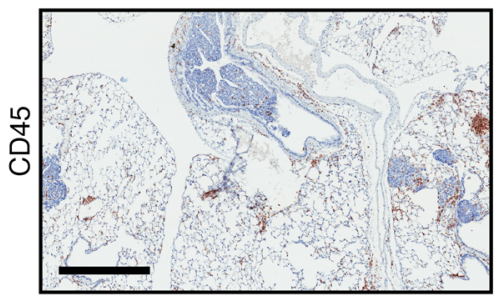

30 days after $\mathrm{PBS}$

gure 4. MYXV localizes to the lungs of tumor bearing mice resulting in a prolonged immune response. (A) Bioluminescence imaging of conditional Ad-Cre-treated $p 53^{-1-} / \mathrm{Rbr}^{-1-} /$

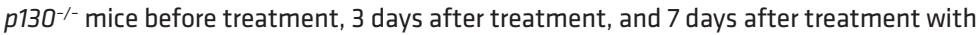
vMyx-FLuc. vMyx-FLuc was administered by intranasal instillation ( $5 \times 10^{7} \mathrm{FFU}$ in $60 \mu \mathrm{lPBS}$ ). (B) Bioluminescent imaging of resected lungs from conditional Ad-Cre-treated $p 53^{-1-} / R b 1^{-1-} /$ p130/- mice (SCLC positive) at 3 and 7 days after treatment with vMyx-FLuc, compared with resected lungs from control mice lacking $p 53 / R 61 / p 130$ knockouts (SCLC negative) 3 days after vMyx-FLuc. (C) Timeline indicating experimental design for examining immune responses 30 days after vMyx-M135KO-GFP. Three months after Ad-Cre tumor induction, mice were treated twice with MYXV $\left(5 \times 10^{7} \mathrm{FFU}\right.$ in $\left.60 \mu \mathrm{PBS}\right) 48$ hours apart via intranasal instillation, 30 days after MYXV treatment mice were euthanized and the lungs examined. (D) Histological analysis of mice 30 days after intranasal instillation of PBS or MYXV showing H\&E-stained FFPE sections. Higher magnification inset shows effects on individual tumorlets. (E) AntiCD45 IHC-stained FFPE serial sections. Scale bars, $500 \mu \mathrm{m}$. monary vMyx-FLuc clearance in vivo by 7 days ( $n=3$ mice) (Figure $4 \mathrm{~A})$. We validated this observation by timed euthanasia after intrapulmonary vMyx-FLuc delivery with detection of luciferase signal exclusively in dissected lungs at 3 days after infection with subsequent viral clearance by 7 days after infection (Figure 4B). We did not detect measurable luciferase in nonpulmonary organs or in PBS-treated mice, indicating that normal noncancerous tissues are essentially nonpermissive for MYXV replication (Supplemental Figure 6).

To determine whether MYXV treatment increases immune cell infiltration in lung tissue and whether it can trigger tumor necrosis, we treated SCLC GEMM mice with 2 doses 48 hours apart of vMyx-GFP-TdT at $5 \times 10^{7} \mathrm{FFU}$ by intranasal instillations starting 3 months after intratracheal Ad-Cre delivery (scheme in Figure 4C). Mice were euthanized 30 days after the second viral treatment and subjected to necropsy. H\&E stained lung sections examined by a masked veterinary pathologist showed increased frequency of scattered discrete foci of necrosis within tumorlets in lung sections from mice treated with vMyx-M135KO-GFP compared with PBS-treated controls (Figure 4D). In addition, we detected a marked increase in CD $45^{+}$ immune cell infiltration of lung tissue surrounding tumorlets only in MYXV-treated mice compared with PBS controls, suggesting an enhanced immunostimulatory response following MYXV infection (Figure 4E).

Intrapulmonary MYXV alone or combined with low-dose cisplatin prolongs overall survival of immunocompetent SCLC GEMM mice. To test the efficacy of MYXV oncolytic therapy to prolong survival of SCLC GEMM mice, we performed a survival study (scheme in Figure 5A) testing intrapulmonary vMyx-M135KO-GFP alone ( 2 doses of $5 \times 10^{7} \mathrm{FFU}$ given 38 hours apart, $n=31$ ) or in combination with low-dose cisplatin $(2.5 \mathrm{mg} / \mathrm{kg}, n=29)$ compared with PBS-alone $(n=31)$ or cisplatin-alone $(n=30)$ controls. Cisplatin was administered by i.p. injection in 4 cycles by treating animals once a week for 3 weeks then allowing 2 weeks rest before starting another cycle (Figure 5A). We observed a statistically significant increase in survival in mice treated with vMyx-M135KO-GFP alone compared with those treated with PBS $(P=0.0016$, median survival 217 days versus 149 days) (Figure 5B). Although high-dose cisplatin is an effective cytotoxic agent for neuroendocrine lineage tumors, we selected lowdose cisplatin treatment cycles and did not observe a statistically significant effect on survival used as a single agent when compared with PBS control $(P=$ 0.78, median survival 161.5 days versus 149 days). In contrast, we observed that the combination of low-dose cisplatin plus MYXV showed a striking 


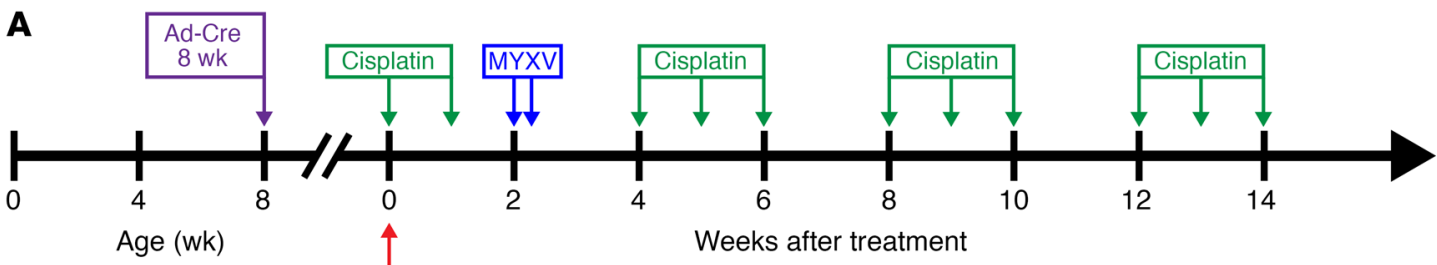

Treatment start 100 days after Ad-Cre

B

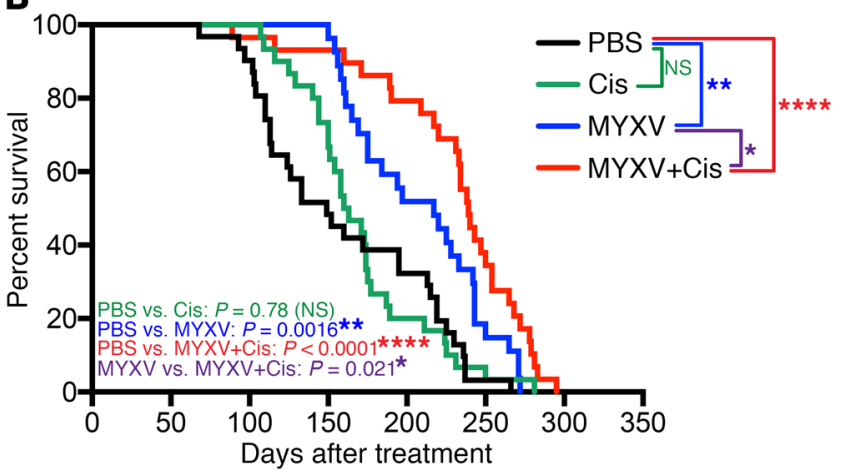

Figure 5. MYXV increases overall survival in conditional $p 53^{-/-/ R b 1^{-1-} /}$ p130-/- SCLC GEMM. Survival is enhanced when MYXV is combined with lowdose cisplatin. (A) Timeline for survival experiment indicating time of Ad-Cre tumor induction at 6-8 weeks of age, treatment start at 100 days after tumor induction, and the indicated time points for the therapeutic intervention. Mice receiving vMyx-M135KO-GFP were given 2 doses $\left(5 \times 10^{7} \mathrm{FFU}\right.$ in 60 $\mu \mathrm{l}$ PBS) 48 hours apart, and cisplatin was administered by i.p. injection (2.5 $\mathrm{mg} / \mathrm{kg}$ in $100 \mu \mathrm{l} \mathrm{PBS}$ ) at the indicated time points. (B) Kaplan-Meier survival analysis of the experiment outlined in Figure 3A. Conditional $p 53^{-1-} / R b 1^{-1-} /$

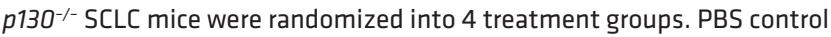
mice received intranasal instillation and i.p. injections of PBS. Mice treated with MYXV alone received i.p. injections of PBS at the same cisplatin treatment intervals, and the cisplatin-only group received intranasal instillation of PBS at the MYXV treatment interval. Survival data represent 31 mice in the PBS group, 30 mice in the cisplatin (Cis) group, 27 mice in the MYXV group, and 29 mice in the MYXV+Cis group. ${ }^{*} P<0.05,{ }^{* *} P<0.01$, ${ }^{* * *} P$ $<0.0001$, NS indicates $P>0.05$, by log-rank (Mantel-Cox) test. Median survival for each treatment group was as follows: PBS: 149 days $(95 \% \mathrm{Cl}$, $113-213$ days); Cis: 161.5 days ( $95 \% \mathrm{Cl}, 150-175$ days); MYXV: 217 days $(95 \% \mathrm{Cl}$, 169-243 days); MYXV+Cis: 239 (95\% Cl, 220-254 days).

of vMyx-FLuc showed the virus was able to replicate within the xenograft tumor in vivo. The viral signal was detectable at 4 and 7 days after intratumoral injection and subsequently at a lower intensity at 10 days (Figure 6C). To determine the effect on tumor histology within the first week, we sacrificed vMyx-FLuc-treated mice and examined tumor histology at 7 days after treatment. We observed extensive necrosis within sections of MYXV-treated mice whereas control tumors treated with intratumoral PBS showed minimal $(<5 \%)$ necrosis $(n=6$ and representative section using ImageJ analysis for percentage of necrosis shown in Figure 6D).

Intratumoral delivery of MYXV to the syngeneic immunocompetent murine SCLC model induces extensive tumor necrosis with marked host immune cell infiltration. We expanded these findings by studying intratumoral MYXV delivery to additional immunodeficient animals carrying SCLC PDX tumors to compare the efficiency of viral clearance using an immunocompetent murine SCLC allograft model. We first generated SCLC allograft tumors in syngeneic immunocompetent mice using independent murine SCLC cell lines derived from our GEMM. These subcutaneous allograft (SQA) tumors were injected intratumorally with either vMyx-FLuc or PBS control (Figure 7A). We observed accelerated clearance of the MYXV luciferase signal in immunocompetent mice by 5 days $(n=4)$ compared with either the immunodeficient human PDX $(n=3)$ (Figure 7B) or the immunodeficient murine SCLC xenograft in NSG mice (Supplemental Figure 10), suggesting a role for immune cell infiltration in targeting virally infected cells. We observed widespread necrosis of SCLC tumor cells within the syngeneic tumor allograft 7 days after intratumoral injection of vMyxFLuc, which was associated with marked $\mathrm{CD} 45^{+}$immune cell infiltration as compared with PBS control intratumoral injections
6) for testing of intratumoral injection and maintenance of the new human SCLC PDX model. Direct intratumoral injections 
A
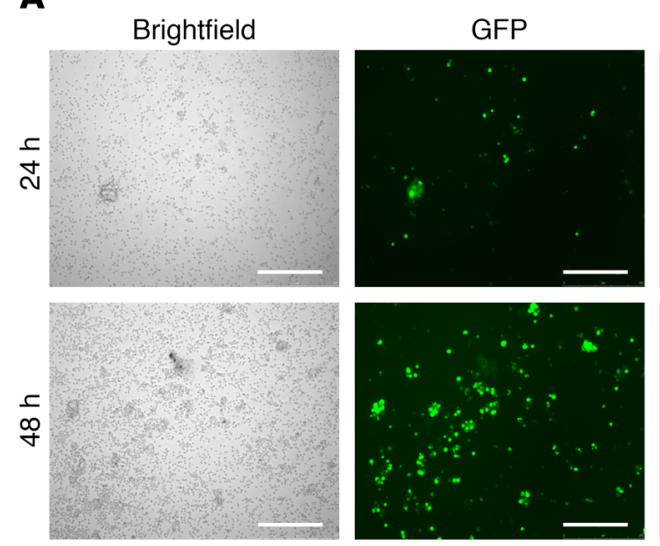

\section{C}
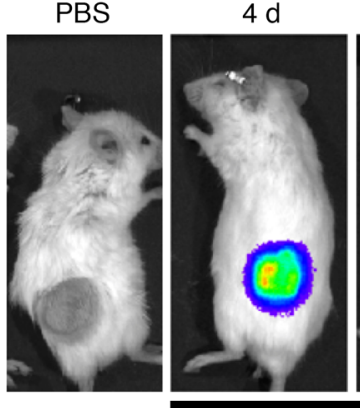

$7 \mathrm{~d}$

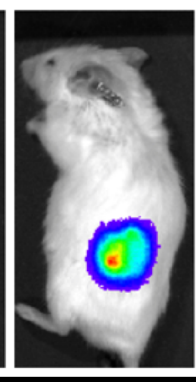

vMyx-FLuc
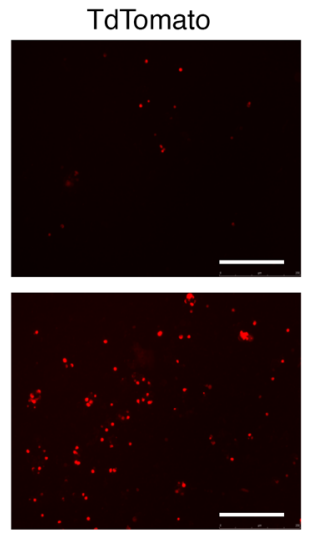

D
B

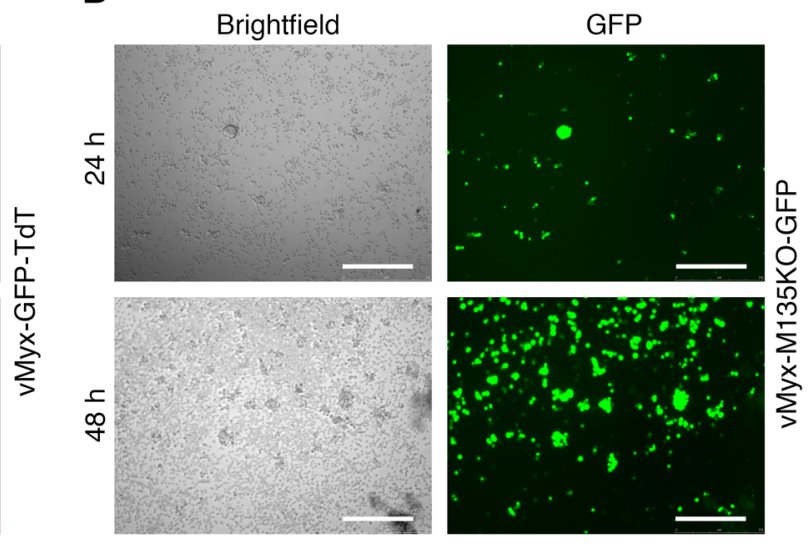
$10 d$

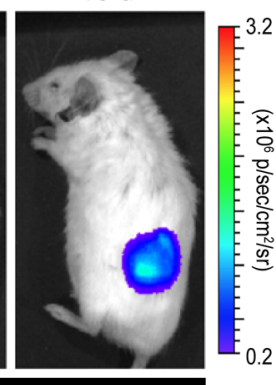

0.2

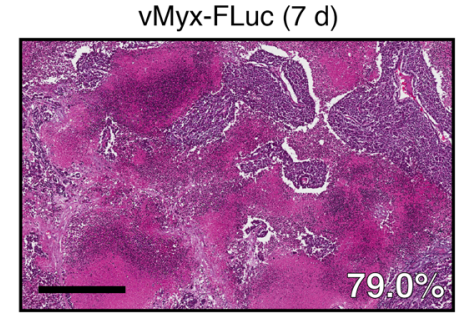

$\operatorname{PBS}(7 \mathrm{~d})$

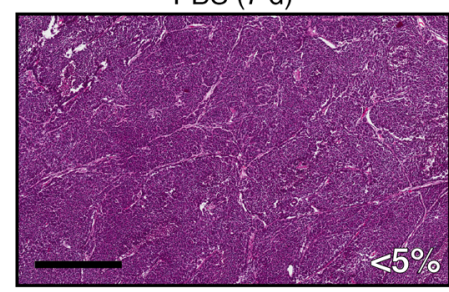

vMyx-M135KO-GFP (7 d)

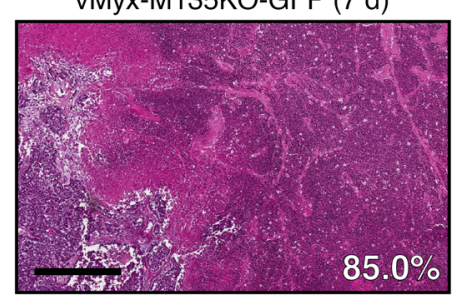

Figure 6. Primary human SCLC specimen is permissive to MYXV, and a direct intratumoral delivery of MYXV to human SCLC PDX tumors shows viral replication and extensive tumor necrosis. (A) Primary SCLC patient specimen collected from bronchoscopy was immediately infected with vMyx-GFP-TdT at $10 \mathrm{MOI}$, demonstrating both early (GFP) and late (TdTomato) gene expression for replication at 48 hours. Scale bars, $200 \mu \mathrm{m}$. Early infection is evident at 24 hours and replication propagating through the patient specimen is evident by 48 hours. (B) Primary SCLC patient specimen infected with vMyx-M135KOGFP at $10 \mathrm{MOI}$ shows expression of early/late (GFP) gene expression at 48 hours. Scale bars, $200 \mu \mathrm{m}$. vMyx-M135KO-GFP lacks a TdTomato reporter gene and expression of the GFP reporter gene is under a poxvirus synthetic early/late promoter expressing GFP during both infection and replication stages. (C) Patient-derived xenograft (PDX) tumor after direct intratumoral injection ( $5 \times 10^{7} \mathrm{FFU}$ in $\left.50 \mu \mathrm{lPBS}\right)$ of vMyx-FLuc expressing Firefly luciferase under poxvirus synthetic early/late promoter indicating viral infection and replication. (D) Histological analysis of H\&E stained FFPE sections from PDX tumors at 4 and 7 days after vMyx-FLuc injection ( $5 \times 10^{7} \mathrm{FFU}$ in $50 \mu \mathrm{lPBS}$ ) showing progressively increasing necrosis. vMyx-M135KO-GFP infection illustrates similar necrotic effects as vMyx-FLuc at 7 days. Scale bars, $500 \mu \mathrm{m}$. Percentage of tissue area showing necrosis is indicated in each micrograph.

(Figure 7C). This result suggests that intratumoral MYXV injection in patients with lung parenchymal or regional chest lymph nodes would induce localized tumor necrosis associated with host immune cell infiltration. To confirm this observation, we delivered intratumoral MYXV or control PBS to a new set of allograft tumorbearing mice and observed that MYXV significantly delayed tumor growth (Figure 7, D and E). We measured viral clearance from the allograft tumors and observed no effect on animal health or body weight (Supplemental Figure 8). We also tested ex vivo exposure of SCLC tumor cells to either MYXV or PBS control and observed complete loss of tumor development following treatment with MYXV in the allograft immunocompetent model (Figure 7F). This ex vivo exposure to MYXV was also examined in an immunodeficient NSG mouse model, where a similar inhibition of tumor growth was observed (Supplemental Figure 9).

\section{Discussion}

There have been few oncolytic virotherapies tested for the treatment of lung cancer and these have focused on mesothelioma and non-SCLC (47). Since the clinical trial testing of Seneca Valley Virus (SSV001) after chemotherapy in patients with advanced SCLC was terminated due to lack of efficacy (ClinicalTrials.gov, NCT01017601), there have been few studies testing oncolytic virotherapy in this important lung cancer subtype. However, this negative clinical trial might have been predicted, since SSVO01 has selective tropism for the rare variant SCLC phenotype (45), while it is nonpermissive for viral replication and cytotoxicity in the more common ASCL1-positive SCLC tumors characterized by in vitro growth as tightly packed floating spheroids. We have now identified a modified MYXV backbone with a high safety index for normal mammalian cells, which shows efficient late 
A<smiles>[GeH2]</smiles>

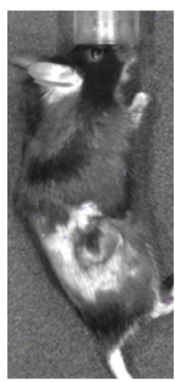

$2 d$

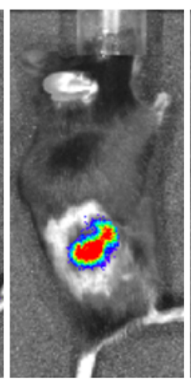

$4 d$

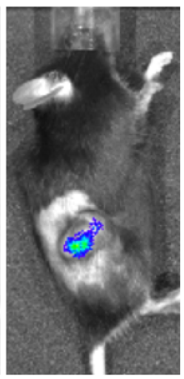

$7 d$

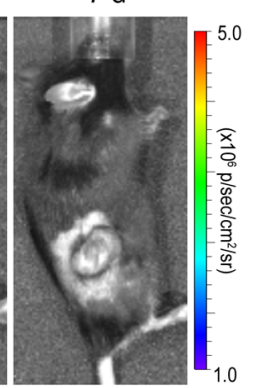

B

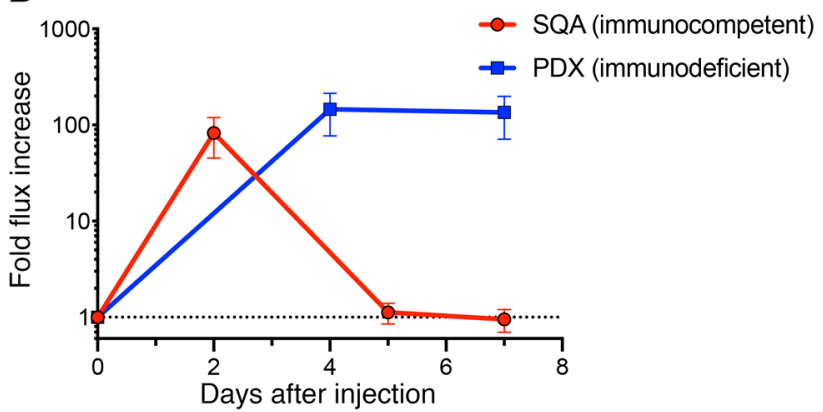

C
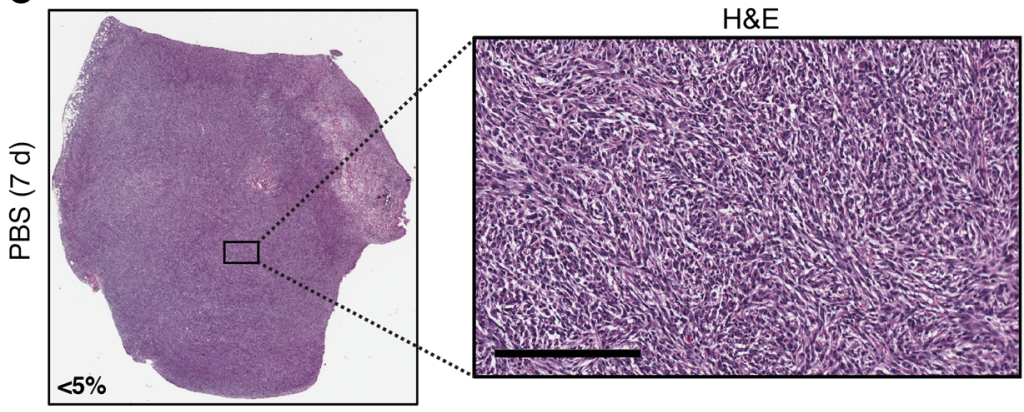

H\&E
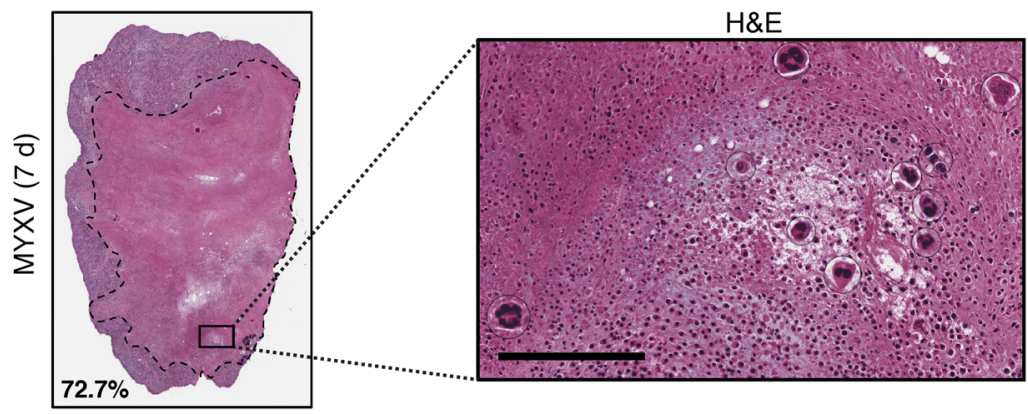
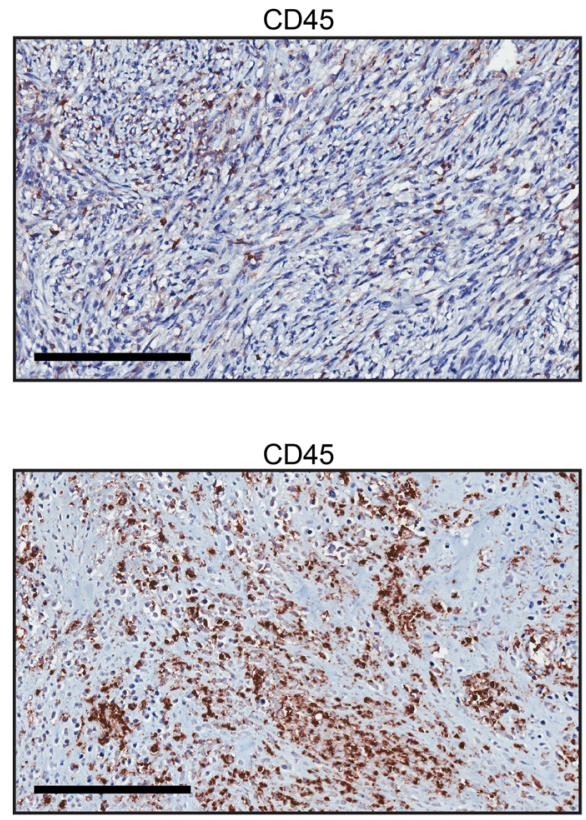

D

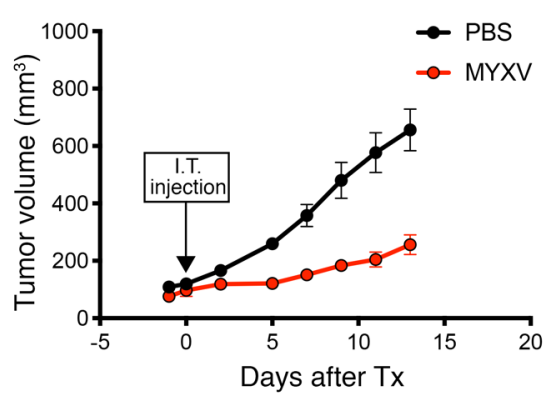

E

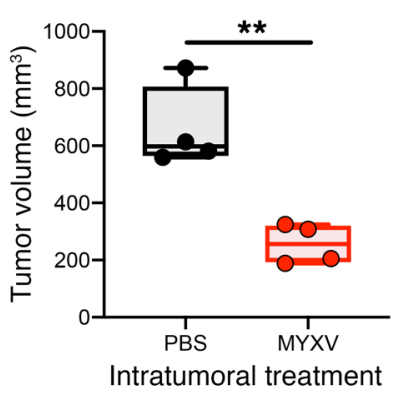

$\mathbf{F}$

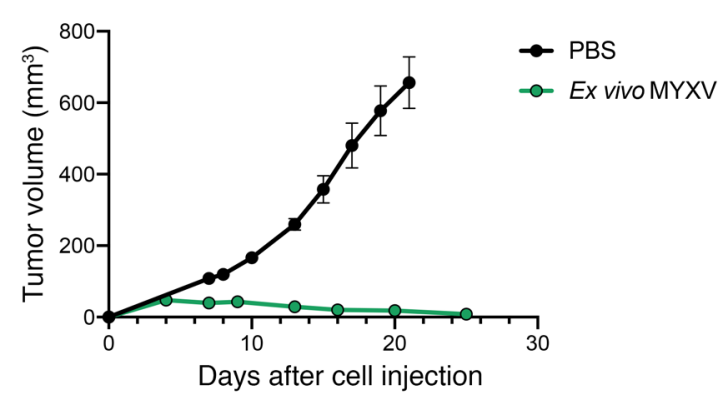

Figure 7. MYXV infection inhibits tumor development and Intratumoral delivery of MYXV to murine syngeneic SCLC allografts show necrosis, immune cell infiltration, and tumor growth inhibition. (A) Subcutaneous allograft (SQA) SCLC tumor in an immunocompetent mouse showing replication and rapid clearance by 7 days after vMyx-FLuc treatment. (B) Quantification of the fold increase in vMyx-FLuc bioluminescence signal from SQA tumors in immunocompetent mice $(n=4)$ compared with PDX tumors in immunodeficient NSG mice $(n=3)$ following direct intratumoral injection of MYXV (vMyxFLuc, $5 \times 10^{7} \mathrm{FFU}$ in $50 \mu \mathrm{lPBS}$ ). Data represent mean \pm SD. (C) Histological analysis of representative H\&E-stained FFPE sections from SQA tumors 7 days after intratumoral injection of vMyx-FLuc $\left(5 \times 10^{7} \mathrm{FFU}\right.$ in $\left.50 \mu \mathrm{lPBS}\right)$ and 7 days after PBS injection ( $50 \mu \mathrm{l}$ PBS). Extensive necrosis is observed in representative MYXV-treated tumor. Higher magnification illustrates the necrotic debris field with select immune cells further enlarged to observe nuclear morphology. Anti-CD45 IHC confirms high levels of immune cell infiltration following MYXV treatment. Scale bars, $200 \mu \mathrm{m}$. ( $\mathbf{D}$ and $\mathbf{E}$ ) Effect of either intratumoral injection of MYXV (vMyx-FLuc, $5 \times 10^{7} \mathrm{FFU}$ in $50 \mu \mathrm{lPBS}$ ) or PBS (50 $\left.\mu \mathrm{l}\right)$. (D) Effect on tumor volume over time ( $n=4$ mice in each group). Data represent mean \pm SEM. (E) Final tumor volume at experimental endpoint ( $n=4$ mice in each group). Box-and-whisker plot showing the minimum to maximum with all data points and the horizontal line representing the median. ${ }^{* *} P<0.01$, by unpaired Student's $t$ test. (F) Murine SCLC cell line (737274-A) infected with vMyx-M135KO-GFP for 24 hours at $10 \mathrm{MOI}$ (ex vivo infection) was injected into immunocompetent mice $(n=3)$ and monitored for tumor development then compared with control PBS-treated tumor development $(n=4)$. Data represent mean \pm SEM. 
viral replication and cytotoxicity in all human and mouse SCLC tumor lines as well as freshly collected human SCLC samples and PDX models. In addition, we observed a statistically significant prolongation of overall survival ( $P=0.0016$; PBS control versus MYXV) in a conditional SCLC GEMM system using intrapulmonary delivery with 2 intranasal MYXV instillations 48 hours apart. Since it is unlikely that intrapulmonary delivery would achieve a sufficiently high MOI for the ablative tumor cytotoxicity to sustain a meaningful impact on overall SCLC GEMM survival, and since we observed that MYXV delivered i.p. did not localize to subcutaneous allograft tumors (Supplemental Figure 10, A and B), we hypothesized that improvement in survival would require intratumoral delivery with induction of host immune cell defenses. We confirmed this hypothesis by detection of enhanced infiltration of immune cells in the immunocompetent GEMM and also following direct intratumoral injections using syngeneic mice carrying allograft SCLC tumors. These data support observations from a recent phase I clinical trial where the tumor response rate and attainment of durable antitumor efficacy using concurrent anti-PD1 immune checkpoint inhibition plus a modified herpes viral vaccine (talimogene laherparepvec) were dependent on the ability of the virotherapy to induce acute host immune cell infiltration (16). These experiments also support reports showing efficacy of proinflammatory intratumoral Maraba virotherapy to activate host immune cell infiltration in triple-negative breast cancer prior to surgical resection (17). Recent studies using intravenous instead of intratumoral oncolytic reovirus delivery also demonstrated induction of cytotoxic $\mathrm{T}$ cell infiltration prior to resection of brain tumors (48). In addition, other groups have tested the potential of intratumoral immune-enhancing agents to induce a systemic anticancer effect. For example, a recent report observed that multiple intratumoral injection of combined unmethylated (cytidine guanine) CG-enriched oligodeoxynucleotides/TLR9 ligand with anti-OX40 antibodies triggered an immune response that was effective in preventing different tumor subtypes in an animal model (49). We also tested the ability of a single intratumoral MYXV to induce a systemic antitumor antisera in an immunocompetent allograft tumor model. We detected tumor reactive serum antibodies in 3 of 4 MYXV-treated mice; however, we also observed tumor reactive serum antibodies in 2 of 4 control mice, perhaps associated with the rapid tumor growth with ulceration detected in PBS-treated allografts (Supplemental Figure 8D). While there have been numerous studies to quantitate the incidence and prognostic significance of de novo immune cell infiltration in non-SCLC $(50,51)$, there are fewer reports in human SCLC since these patients rarely undergo surgical resection. One study in patients with advanced SCLC noted that low tumorassociated $\mathrm{CD} 45^{+}$counts were the best predictor of poor clinical outcome despite good performance status and other favorable clinical biomarkers (52). In addition, a large study of patients with SCLC noted that anergy toward skin hypersensitivity testing was a strong predictor for shortened survival, especially in the subset of otherwise healthy patients with lower tumor burden (53). We tested 26 new human SCLC tumor samples and found scant immune cell infiltration. These data, as well as other reports showing reduced prevalence of infiltrating lymphocytes in SCLC compared with non-SCLC $(52,54)$, may limit the potential therapeutic benefit with immune checkpoint inhibition (7) and may correlate with the limited role of PD-L1 as a biomarker for immunotherapy in SCLC (13). Our interventional pulmonary group has shown that intratumoral delivery of anticancer agents to localized lung and regional nodal metastases using endoscopic ultrasound is feasible $(55,56)$. The extensive necrosis observed following intratumoral injection of MYXV is predicted to release SCLC tumor-associated antigens. In addition, the rapid clearance of MYXV associated with sustained immune cell infiltration suggests that MYXV-infected SCLC undergoes immunologic cell death in our immunocompetent models. Experiments showing the efficacy of MYXV to induce immune cell infiltration and prolong survival support the testing of intratumoral MYXV therapy and warrant further investigation of MYXV combined with immune checkpoint inhibition in SCLC.

\section{Methods}

Genetic mouse models and tumor induction. The $\mathrm{p} 53^{\operatorname{lox} / \operatorname{loxp}} \mathrm{p} 130^{\operatorname{lox} 2722 / 10 \times 2722}$ $\mathrm{Rb}^{\text {loxP/loxp }}$ mouse strain has been previously described (46) and was provided by Julien Sage (Stanford Cancer Center, Palo Alto, CA). Presence of floxed sequences (loxP and lox2722) in GEMMs was confirmed by PCR. Genomic DNA was extracted using the Qiagen Blood \& Tissue Analysis kit (catalog 69506), and PCR was performed using Illustra PuReTaq Ready-to-go PCR beads (GE Healthcare, 27-9557-02) with primers previously reported $(46,57-60)$. Tumors were initiated when mice were 6-8 weeks of age by intratracheal injection of adenovirus serotype 5 expressing Cre-recombinase fused to enhancer GFP under a CMV promoter (Ad5CMVCre-eGFP, University of Iowa Vector Core, catalog WC-U of Iowa 1174) as previously described (61). Intranasal instillation of MYXV was performed 100 days after Ad-Cre delivery, which was the timing previously determined by pilot experiments (shown in Figure 2B) to be required for the onset of SCLC tumorlets. Survival events were scored when the Body Condition Score (as defined by the American Association for Laboratory Animal Science) (62) of animals declined or per absolute survival events. Mice were maintained within the University of Florida Cancer Genetics Research Center and Communicore barrier facilities.

Patient-derived xenograft and murine allograft tumor models. Tissue samples were collected from patients at the time of transbronchial biopsy at the University of Florida Health Hospital following informed consent under IRB approval. Lesions were located using endobronchial ultrasound (EBUS), and patient samples used for PDX studies were confirmed to be SCLC by an independent pathologist. Patient samples were transported in DMEM lacking FBS on ice and aliquoted for in vitro testing as well as immediately implanted subcutaneously in an NSG (NOD.Cg-Prkdc ${ }^{\text {scid }}$ Il2 $\mathrm{rg}^{\text {tmlwil }} /$ SzJ strain) mouse (passage $\mathrm{P}_{\mathrm{o}}$ ). Once the PDX tumor reached $1.5 \mathrm{~cm}$ in diameter it was excised, minced using a scalpel, and implanted subcutaneously in additional NSG mice (passage $\mathrm{P}_{1}$ ). Once tumors reached $8-10 \mathrm{~mm}$ in diameter they were randomized into treatment groups or passage to maintain the tumor in NSG mice. Treatment animals received intratumoral injections of vMyx-FLuc $\left(5 \times 10^{7} \mathrm{FFU}\right.$ in $\left.50 \mu \mathrm{l}\right)$ or PBS $(50 \mu \mathrm{l})$. To maintain the PDX, tumors were excised and either minced using a scalpel and cryogenically preserved in freezing medium (70\% RPMI-1640, $20 \%$ FBS, 10\% DMSO) or minced using a scalpel and implanted subcutaneously in additional NSG mice (passage $\mathrm{P}_{2}$ ). Once passage 
$\mathrm{P}_{2}$ tumors reached 8-10 $\mathrm{mm}$ in diameter they were randomized into treatment groups receiving either vMyx-FLuc $\left(5 \times 10^{7} \mathrm{FFU}\right.$ in $\left.50 \mu \mathrm{l}\right)$, vMyx-M135KO-GFP $\left(5 \times 10^{7} \mathrm{FFU}\right.$ in $\left.50 \mu \mathrm{l}\right)$, or PBS $(50 \mu \mathrm{l})$.

Murine subcutaneous allograft tumors were generated using murine SCLC cell lines derived from single lung tumor foci in p53 $3^{\text {loxp/loxp }}$ p130 ${ }^{\text {lox2722/lox2722 }} \mathrm{Rb}^{\text {loxP/loxp }}$ mice after treatment with Ad-Cre. Cell lines were confirmed by PCR to have the designed conditional $\mathrm{p} 53^{-/-} / \mathrm{p} 130^{-/-} / \mathrm{Rb}^{-/-}$gene knockouts. Cell lines were then injected subcutaneously into 6- to 8 -week-old $\mathrm{p} 53^{\operatorname{loxP} / \operatorname{loxP}} \mathrm{p} 130^{\text {lox2722/lox2722 }}$ $\mathrm{Rb}^{\text {loxP/loxp }}$ mice that were not given Ad-Cre, but served as matched genetic background recipients for the cell lines. The mouse tumor cell lines tested in this model were collected from in-bred generation number 14 animals and breeding was performed in the University of Florida animal facility. The resulting allograft tumors were treated with either vMyx-FLuc $\left(5 \times 10^{7} \mathrm{FFU}\right.$ in $\left.50 \mu \mathrm{l}\right)$ or PBS $(50 \mu \mathrm{l})$. All intratumoral injections were performed using a 27-gauge needle. Murine SCLC subcutaneous tumors generated in NSG mice were treated with vMyx-FLuc $\left(5 \times 10^{7} \mathrm{FFU}\right.$ in $\left.50 \mu \mathrm{l}\right)$ by intratumoral injection or intraperitoneal injection, and 15 days after MYXV treatment the total tumor titer was determined from titering whole-tumor homogenates on permissive RK13 cells.

Cell sources, propagation, and imaging. All human SCLC cells were obtained from the original NCI-Navy Oncology stock (63), validated by genotyping, and grown in RPMI-1640 culture media (Sigma-Aldrich, catalog R8758) supplemented with 10\% FBS (Invitrogen Gibco, catalog 26140-079). Murine GEMM-derived SCLC cell lines were generated from $\mathrm{p} 53^{\text {loxP/loxP }} \mathrm{p} 130^{\text {lox2722/lox2722 }} \mathrm{Rb} \mathrm{b}^{\text {loxp/loxP }}$ mice by excision of solitary 1-mm tumor foci arising in lungs and established in HITES growth medium (RPMI-1640, 5\% FBS, hydrocortisone, insulin, transferrin, estrogen, and selenium) then propagated in RPMI-1640 supplemented with $10 \%$ FBS (cell lines 1.1A, $1.5 \mathrm{~A}, 2.1 \mathrm{~A}, 2.2 \mathrm{~A}, 2.4 \mathrm{~A}, 1.3 \mathrm{~F}, 1.4 \mathrm{~F}$ ) or established and propagated in only RPMI-1640 supplemented with 10\% FBS (cell line 737274-A). We studied a total of 6 different human SCLC cell lines and 8 murine SCLC lines. Normal human bronchial epithelial (NHBE) cells were purchased from Lonza. RK13 cell line was purchased from ATCC (CCL-37). BSC40 cell line was purchased from ATCC (ATCC CRL-2761). NHBE cells were cultured in bronchial epithelial growth medium (BEGM) media (Lonza biotech CC-3170). MEF, RK13, and BSC40 cell lines were cultured in DMEM supplemented with $10 \% \mathrm{FBS}, 100 \mathrm{U} / \mathrm{ml}$ penicillin, and $100 \mu \mathrm{g} / \mathrm{ml}$ streptomycin. All cell lines and assay cultures were grown at $37^{\circ} \mathrm{C}$ with $5 \% \mathrm{CO}_{2}$ and tested to exclude mycoplasma contamination (Venor GeM Mycoplasma Detection Kit, MilliporeSigma). A cisplatin-resistant murine SCLC cell line (2.1A-CR) was generated by continuous exposure to cisplatin with periodic high concentration cisplatin pulses to generate resistant clones, and viability curves performed with the cisplatin-resistant cell line (2.1A-CR) were compared with an equal passage cisplatin treatment-naive parental cell line (2.1A). Viability assays were performed using CellTiter Blue (Promega) following a 72-hour incubation with increasing cisplatin concentrations. Ex vivo MYXV infections were performed at 10 MOI for 24 hours, infection was confirmed by fluorescence microscopy, and cells were then collected, counted, and washed once in PBS before $5 \times 10^{6}$ cells were injected subcutaneously into the right flank.

Myxoma virus infection, fluorescence imaging, replication of viral progeny, immunogenic cell death assay. Stocks of Myxoma viruses were grown using RK13 or BSC40 cells and purified by centrifugation on a sucrose gradient. Constructions of wild-type MYXV (WT-MYXV) expressing different reporter proteins vMyx-GFP-TdT (WT-MYXV expressing GFP under a poxvirus synthetic early/late promoter and TdTomato under a poxvirus p11 late promoter); vMyx-FLuc (WT-MYXV expressing the Firefly luciferase protein [FLuc] under a poxvirus synthetic early late promoter) and vMyx-M135KO-GFP (a recombinant MYXV with deletion of the M135 gene and by insertion of GFP under a poxvirus synthetic early/late promoter) were described previously (64). To examine the production of viral progeny, murine SCLC cells were seeded in 24-well plates at $5 \times 10^{4}$ cells per well and after overnight attachment were infected with vMyx-GFP$\mathrm{TdT}$ at a MOI of 1 and harvested at 1 hour (to measure input virus) and also either 24,48 , or 72 hours after infection (to measure progeny virus formation). MYXV virus production was titered on permissive RK13 cells. For ATP release assays (42), cells were seeded in opaque-walled 96-well assay plates (Corning Inc.) at 10,000 cells/well (100 $\mu \mathrm{l})$. Cells were allowed to adhere or normalize for 12-16 hours and then they were infected with either vMyx-GFP or vMyx-M135KO-GFP at a MOI of 10. Cells were incubated according to their culture condition. At the indicated time points, an equal volume $(100 \mu \mathrm{l})$ of CellTiter-Glo reagent (Promega Corporation) was added to each well. The contents were mixed for 2 minutes on an orbital shaker for cell lysis. The cells were then incubated at room temperature for 10 minutes to stabilize luminescence signal. Luminescence signal was recorded using a Varioskan Lux microplate reader (Thermo Fisher Scientific).

Electron microscopy. Human SCLC cell line $\mathrm{H} 60$ was infected with vMyx-M135KO-GFP at a MOI of 10. After 1 hour of infection, the cells were collected by centrifugation at $600 \mathrm{~g}$ for 5 minutes and resuspended in media. At 48 hours after infection, cells were harvested by centrifugation at $600 \mathrm{~g}$ and resuspended in $4 \mathrm{ml}$ cacodylate buffer ( $0.1 \mathrm{M}$ cacodylate $\mathrm{pH} 7.2,2 \mathrm{mM} \mathrm{MgCl}_{2}, 1 \mathrm{mM} \mathrm{CaCl}_{2}, 43 \mathrm{mM} \mathrm{NaCl}$ ). Cells were collected by centrifugation at $600 \mathrm{~g}$ and resuspended in $2 \mathrm{ml}$ of $2 \%$ glutaraldehyde in cacodylate buffer. The cells were incubated at room temperature for 1 hour with occasional rocking and kept at $4^{\circ} \mathrm{C}$ before further processing. Cells were then collected by centrifugation and post-fixed in $1 \%$ osmium tetroxide water. After dehydration with increasing concentrations of ethanol solution, followed by $100 \%$ acetone, the cells were embedded in Spurr resin mix (Sigma Aldrich). Following thin sectioning, the samples were post-stained with uranyl acetate and Reynolds lead acetate and examined on an FEI Spirit 120kV transmission electron microscope (FEI company subsidiary of Thermo Fisher), with the assistance of the University of Florida ICBR Electron Microscopy Core Laboratory.

Bioluminescence imaging. All mice were shaved prior to bioluminescence imaging on a Xenogen IVIS Lumina Bioluminescence Imaging System (Perkin-Elmer). Mice were anesthetized with 2.5\% isoflurane in $\mathrm{O}_{2}$ and imaged in a XIC-3 Animal Isolation Chamber (Perkin-Elmer, cat$\operatorname{alog} 123997)$ at the peak of luciferase activity (10 minutes after injection, 10-second exposure setting). Mice were injected i.p. with D-luciferin (150 mg/kg in PBS). Total photon flux read was analyzed and restricted to tumor ROI on Living Image v2.60.1 software (Imaging Systems).

Histology and immunohistochemistry. Tissue specimens were fixed in $4 \%$ formalin for 24 hours and stored in $70 \%$ ethanol prior to paraffin processing and embedding. Tissue sections $(5 \mu \mathrm{m})$ were used for $\mathrm{H} \& \mathrm{E}$ staining (Richard Allan Scientific, catalog 7211) or for IHC staining. Prior to IHC staining, sections were deparaffinized and the endogenous peroxidase activity was blocked. Antigen retrieval was performed 
using citrate buffer. Antibodies used for staining include anti-CD45 (BD Biosciences, catalog 550539, clone 30-F11), anti-CD56/NCAM (Millipore, catalog AB5032), anti-CD3 (Abcam, catalog ab16669, clone SP7). Sections were developed with DAB and counterstained with hematoxylin. Automated image acquisition was performed using an Aperio Scanscope CS Slide Scanner system with a $\times 20$ objective (Aperio Technologies). In addition, a tissue microarray (TMA) was constructed with 1.0-mm cores from 26 small-cell cancer specimens embedded in formalin-fixed, paraffin-embedded (FFPE) tissue. The TMA was constructed utilizing standard equipment (Beecher Instruments). To create the TMA, H\&E slides with SCLC were reviewed by a pathologist to select FFPE tissue blocks with adequate tumor, and the tumor areas were annotated on the slides. A core sample was removed from each block in an area corresponding to the specified tumor area in the H\&E slide. Each core was embedded in a SCLC TMA paraffin block according to a map.

The TMA was stained to identify lymphocytes with CD45 IHC. IHC was performed in the Moffitt Cancer Center Tissue Core Facility using a Ventana Discovery XT automated system (Ventana Medical Systems), using a rabbit primary anti-CD45 antibody (Abcam, cata$\log$ ab10558, 1:200 dilution) as previously described (65). Briefly, the slide was deparaffinized on the automated system with EZ Prep solution (Ventana), heat-induced antigen retrieval was performed, and the specimen was incubated with the anti-CD45 antibody diluted in Dako antibody diluent for 32 minutes. The Ventana OmniMap AntiRabbit Secondary Antibody was incubated for 8 minutes and signal was detected using the Ventana ChromoMap kit. The slide was then counterstained with hematoxylin, dehydrated, and coverslipped.

The CD45-stained SCLC TMA was then evaluated for CD $45^{+}$ lymphocytes by a board-certified pathologist. The SCLC specimens were scored using a 4-tier immunoscore with a scale from 0-3 based on evaluation of the percentage of lymphocytes in the stroma, as previously described (66). With this strategy, 0 represents 0-5\% lymphocytes in the stroma (negative to minimal), 1 represents $6-25 \%$ (mild), 2 represents $26-50 \%$ (marked), and 3 represents greater than $50 \%$ (severe).

Flow cytometry. Lung tissue single-cell suspensions were prepared immediately following euthanasia by carbon dioxide asphyxiation. The lung was excised and inflated with digest solution containing 1.5 $\mathrm{mg} / \mathrm{ml}$ collagenase A (Roche) and $50 \mathrm{U} / \mathrm{ml}$ DNaseI (MilliporeSigma, catalog D4513) in HBSS with 5\% FBS, $10 \mathrm{mM}$ HEPES, and a final calcium concentration of $5 \mathrm{mM}$. The increased final calcium concentration was obtained by addition of concentrated $\mathrm{CaCl}_{2}$. Lungs were then diced into smaller specimens and transferred to an additional 4 $\mathrm{ml}$ of digest solution and incubated at $37^{\circ} \mathrm{C}$ with gentle shaking for 45 minutes and then vortexed for $2-3$ seconds every 5 minutes. Following enzymatic digestion, $30 \mathrm{ml}$ PBS was added and the sample vortexed for 30 seconds before being passed through a $70-\mu \mathrm{m}$ strainer. Samples were then treated with ACK RBC lysis buffer, washed with PBS, counted, and plated at $1 \times 10^{6}$ cells in $100 \mu$, then stained for flow cytometry. Viability staining was performed using LIVE/DEAD Fixable Yellow Dead Cell Stain (Thermo Fisher Scientific, catalog L34959) according to the manufacturer's protocol. Antibody staining was performed using anti-CD45-PE-Cy7 (Biolegend, catalog 103113, clone 30-F11), anti-CD3-V450 (BD Biosciences, catalog 561389, clone 17A2), and anti-CD56-APC (R\&D Systems, catalog FAB7820A, clone 809220). Data were acquired using either a BD LSRII or BD LSRFortessa flow cytometer and the associated BD FACSDiva software (BD Biosciences). Compensation was performed using the associated $\mathrm{BD}$ FACSDiva software at the start of the experiment. Data were analyzed using FlowJo software version 10.4.2.

Chemotherapeutics. Cisplatin was obtained from MilliporeSigma (catalog 479306) and diluted in PBS at $1.25 \mathrm{mg} / \mathrm{ml}$. Mice received 2 treatments with cisplatin by i.p. injection of $2.5 \mathrm{mg} / \mathrm{kg}$ prior to MYXV administration (at 2 weeks and again 1 week prior to MYXV). Following MYXV administration, mice were treated for 3 cycles as follows: for each cycle cisplatin was administered i.p. weekly times 3 , followed by a 2-week break between cycles (Figure 4A).

Randomization of mouse models to treatment groups. Mice were enrolled into alternating treatment groups on a cage-by-cage basis. Different cages of mice from the same litter and from the same parents were distributed across treatment groups as evenly as possible. Histologic lung sections were reviewed by a veterinary pathologist who was masked to the assigned treatment groups.

Statistics. Statistical analyses were performed using the GraphPad Prism 7.0 software. Values are presented as mean \pm SD. Data were analyzed by 2-tailed Student's $t$ test for comparisons between 2 groups, or 1-way ANOVA followed by Tukey's multiple comparisons test to determine significance between multiple groups. Sample size for in vivo experiments was determined using the software $\mathrm{G}^{*}$ Power (v3.1.9.3) with experimental data from preliminary studies to predict the number of animals necessary to give greater than $80 \%$ confidence for a $20 \%$ change in the response variable at the $P<0.05$ significance level. Animals were assigned to treatment groups randomly and the number of animals in each treatment group is reported in the figures. Kaplan-Meier survival curves were analyzed using the log-rank (Mantel-Cox) test to determine significance. For all studies only $P$ values less than 0.05 were considered to have statistical significance.

Study approval. All animal work was conducted under the approval of the University of Florida Institutional Animal Care and Use Committee in accordance with federal, state, and local guidelines. Human tumor specimens were obtained in compliance with state and federal regulation from the University of Florida through an IRB-approved clinical study (IRB26857235) and after obtaining written informed consent from the patients.

\section{Author contributions}

PK, DS, MMR, NM, AN, CH, MZK, and FJK, performed experiments and data analysis. MZK, FJK, GM, PK, DS, MMR, and NM designed the experiments and interpreted the results. MR, CC, TB, SA, MZ, and MVG provided histology interpretation and analyzed histology data. MJ and HJM provided patient samples. MZK, FJK, PK, and DS wrote the manuscript. All authors reviewed the manuscript.

\section{Acknowledgments}

We thank Bertram Jacob (Arizona State University) and Chris Cogle (University of Florida) for helpful scientific advice and for critical reading of the manuscript, and Julian Sage for $\mathrm{p} 53^{\text {loxP/loxP }}$ p130 ${ }^{\text {lox2722/lox2722 }} \mathrm{Rb}^{\text {loxP/loxP }}$ mice. This work was funded by the James and Esther King grant 5JKO4 from Florida Department of Health (to FJK, MZK, GM, and MJ), the Gatorade Trust through funds distributed by the University of Florida, Department of Medicine 
(to MZK and FJK) and in part by Public Health Service grant R01 CA-188132 (to MZK) from the National Institutes of Health, and by an anonymous donation to the University of Florida Health Cancer Center "Janet and I fight back against small-cell lung cancer fund" (to MZK) that initiated this project. This work was supported in part by the Tissue Core Facility at the H. Lee Moffitt Cancer Center \& Research Institute (P30-CA076292).
Address correspondence to: Frederic J. Kaye, University of Florida, Cancer and Genetics Research Complex, 2033 Mowry Road, Room 363, Gainesville, Florida 32610, USA. Phone: 352.273.9152; Email: fkaye@ufl.edu.

MMR and GM's present address is: The Biodesign Institute, Arizona State University, Tempe, Arizona, USA.
1. [No authors listed]. A plan of attack for deadly cancers. Cancer Discov. 2014;4(9):980.

2. Bunn PA, et al. Small cell lung cancer: can recent advances in biology and molecular biology be translated into improved outcomes? J Thorac Oncol. 2016;11(4):453-474.

3. Yang D, et al. Intertumoral heterogeneity in SCLC Is influenced by the cell type of origin. Cancer Discov. 2018;8(10):1316-1331.

4. Harbour JW, Lai SL, Whang-Peng J, Gazdar AF, Minna JD, Kaye FJ. Abnormalities in structure and expression of the human retinoblastoma gene in SCLC. Science. 1988;241(4863):353-357.

5. Takahashi T, et al. p53: a frequent target for genetic abnormalities in lung cancer. Science. 1989;246(4929):491-494.

6. Chute JP, Chen T, Feigal E, Simon R, Johnson BE. Twenty years of phase III trials for patients with extensive-stage small-cell lung cancer: perceptible progress. JClin Oncol.1999;17(6):1794-1801.

7. Antonia SJ, et al. Nivolumab alone and nivolumab plus ipilimumab in recurrent small-cell lung cancer (CheckMate 032): a multicentre, open-label, phase 1/2 trial. Lancet Oncol. 2016;17(7):883-895.

8. Sabari JK, Lok BH, Laird JH, Poirier JT, Rudin CM. Unravelling the biology of SCLC: implications for therapy. Nat Rev Clin Oncol. 2017;14(9):549-561.

9. Doyle A, et al. Markedly decreased expression of class I histocompatibility antigens, protein, and mRNA in human small-cell lung cancer. J Exp Med. 1985;161(5):1135-1151.

10. Yamazaki K, Spruill G, Rhoderick J, Spielman J, Savaraj N, Podack ER. Small cell lung carcinomas express shared and private tumor antigens presented by HLA-A1 or HLA-A2. Cancer Res. 1999;59(18):4642-4650.

11. Yazawa $\mathrm{T}$, et al. Lack of class II transactivator causes severe deficiency of HLA-DR expression in small cell lung cancer. J Pathol. 1999;187(2):191-199.

12. George J, et al. Genomic amplification of CD274 (PD-L1) in small-cell lung cancer. Clin Cancer Res. 2017;23(5):1220-1226.

13. Hellmann MD, et al. Tumor mutational burden and efficacy of nivolumab monotherapy and in combination with ipilimumab in small-cell lung cancer. Cancer Cell. 2018;33(5):853-861.e4.

14. $\mathrm{Yu} \mathrm{H}$, et al. PD-L1 expression by two complementary diagnostic assays and mRNA in situ hybridization in small cell lung cancer. J Thorac Oncol. 2017;12(1):110-120.

15. Suda K, et al. Heterogeneity in immune marker expression after acquisition of resistance to EGFR kinase inhibitors: analysis of a case with small cell lung cancer transformation. J Thorac Oncol. 2017;12(6):1015-1020.

16. Ribas A, et al. Oncolytic virotherapy promotes intra- tumoral $\mathrm{T}$ cell infiltration and improves anti-PD-1 immunotherapy. Cell. 2017;170(6):1109-1119.e10.

17. Bourgeois-Daigneault MC, et al. Neoadjuvant oncolytic virotherapy before surgery sensitizes triple-negative breast cancer to immune checkpoint therapy. Sci Transl Med. 2018;10(422):eaao1641.

18. Chen DS, Mellman I. Elements of cancer immunity and the cancer-immune set point. Nature. 2017;541(7637):321-330.

19. Chiocca EA, Rabkin SD. Oncolytic viruses and their application to cancer immunotherapy. Cancer Immunol Res. 2014;2(4):295-300.

20. Howells A, Marelli G, Lemoine NR, Wang Y. Oncolytic viruses-interaction of virus and tumor cells in the battle to eliminate cancer. Front Oncol. 2017;7:195

21. Ries S, Korn WM. ONYX-015: mechanisms of action and clinical potential of a replicationselective adenovirus. Br JCancer. 2002;86(1):5-11.

22. Seymour LW, Fisher KD. Oncolytic viruses: finally delivering. Br JCancer. 2016;114(4):357-361.

23. Bommareddy PK, Kaufman HL. Unleashing the therapeutic potential of oncolytic viruses. J Clin Invest. 2018;128(4):1258-1260.

24. Andtbacka RH, et al. Talimogene laherparepvec improves durable response rate in patients with advanced melanoma. J Clin Oncol. 2015;33(25):2780-2788.

25. Andtbacka RH, et al. Cutaneous head and neck melanoma in OPTiM, a randomized phase 3 trial of talimogene laherparepvec versus granulocytemacrophage colony-stimulating factor for the treatment of unresected stage IIIB/IIIC/IV melanoma. Head Neck. 2016;38(12):1752-1758.

26. Zamarin D, et al. PD-L1 in tumor microenvironment mediates resistance to oncolytic immunotherapy. J Clin Invest. 2018;128(4):1413-1428.

27. Chan WM, Rahman MM, McFadden G. Oncolytic myxoma virus: the path to clinic. Vaccine. 2013;31(39):4252-4258

28. Villa NY, et al. Ex vivo virotherapy with myxoma virus does not impair hematopoietic stem and progenitor cells. Cytotherapy. 2016;18(3):465-480.

29. Kerr PJ. Myxomatosis in Australia and Europe: a model for emerging infectious diseases. Antiviral Res. 2012;93(3):387-415.

30. Chan WM, McFadden G. Oncolytic poxviruses. Annu Rev Virol. 2014;1(1):119-141.

31. Pisklakova A, et al. M011L-deficient oncolytic myxoma virus induces apoptosis in brain tumor-initiating cells and enhances survival in a novel immunocompetent mouse model of glioblastoma. Neuro Oncology. 2016;18(8):1088-1098.

32. Rahman MM, Bagdassarian E, Ali MAM, McFadden G. Identification of host DEAD-box RNA helicases that regulate cellular tropism of oncolytic Myxoma virus in human cancer cells.
Sci Rep. 2017;7(1):15710.

33. Bell J, McFadden G. Viruses for tumor therapy. Cell Host Microbe. 2014;15(3):260-265.

34. Kaye FJ. RB and cyclin dependent kinase pathways: defining a distinction between RB and p16 loss in lung cancer. Oncogene. 2002;21(45):6908-6914.

35. Cui M, et al. PTEN is a potent suppressor of small cell lung cancer. Mol Cancer Res. 2014;12(5):654-659.

36. Peifer $\mathrm{M}$, et al. Integrative genome analyses identify key somatic driver mutations of small-cell lung cancer. Nat Genet. 2012;44(10):1104-1110.

37. Kim M, et al. The viral tropism of two distinct oncolytic viruses, reovirus and myxoma virus, is modulated by cellular tumor suppressor gene status. Oncogene. 2010;29(27):3990-3996.

38. Wang $G$, et al. Infection of human cancer cells with myxoma virus requires Akt activation via interaction with a viral ankyrin-repeat host range factor. Proc Natl Acad Sci U S A. 2006;103(12):4640-4645.

39. Reck M, Heigener D, Reinmuth N. Immunotherapy for small-cell lung cancer: emerging evidence. Future Oncol. 2016;12(7):931-943.

40. Barrett JW, et al. M135R is a novel cell surface virulence factor of myxoma virus. J Virol. 2007;81(1):106-114.

41. Barrett JW, et al. Identification of host range mutants of myxoma virus with altered oncolytic potential in human glioma cells. J Neurovirol. 2007;13(6):549-560.

42. Kepp O, et al. Consensus guidelines for the detection of immunogenic cell death. Oncoimmunology. 2014;3(9):e955691.

43. Carney DN, et al. Establishment and identification of small cell lung cancer cell lines having classic and variant features. Cancer Res. 1985;45(6):2913-2923.

44. Gazdar AF, Carney DN, Nau MM, Minna JD. Characterization of variant subclasses of cell lines derived from small cell lung cancer having distinctive biochemical, morphological, and growth properties. Cancer Res. 1985;45(6):2924-2930.

45. Poirier JT, Dobromilskaya I, Moriarty WF, Peacock CD, Hann CL, Rudin CM. Selective tropism of Seneca Valley virus for variant subtype small cell lung cancer. J Natl Cancer Inst. 2013;105(14):1059-1065.

46. Schaffer BE, et al. Loss of p130 accelerates tumor development in a mouse model for human small-cell lung carcinoma. Cancer Res. 2010;70(10):3877-3883.

47. Ahmad Z, Kratzke RA. Novel oncolytic viral therapies in patients with thoracic malignancies. Oncolytic Virother. 2017;6:1-9.

48. Samson A, et al. Intravenous delivery of oncolytic reovirus to brain tumor patients immunologically primes for subsequent checkpoint blockade. Sci 
Transl Med. 2018;10(422): eaam757.

49. Sagiv-Barfi I, et al. Eradication of spontaneous malignancy by local immunotherapy. Sci Transl Med.2018;10(426):eaan4488.

50. Brambilla E, et al. Prognostic effect of tumor lymphocytic infiltration in resectable non-small-cell lung cancer. JClin Oncol. 2016;34(11):1223-1230.

51. Bremnes RM, et al. The role of tumor-infiltrating lymphocytes in development, progression, and prognosis of non-small cell lung cancer. J Thorac Oncol. 2016;11(6):789-800.

52. Wang $\mathrm{W}$, et al. Histologic assessment of tumor-associated $\mathrm{CD} 45(+)$ cell numbers is an independent predictor of prognosis in small cell lung cancer. Chest. 2013;143(1):146-151.

53. Johnston-Early A, et al. Delayed hypersensitivity skin testing as a prognostic indicator in patients with small cell lung cancer. Cancer. 1983;52(8):1395-1400.

54. Kim HS, et al. Association of PD-L1 expression with tumor-infiltrating immune cells and mutation burden in high-grade neuroendocrine carcinoma of the lung. J Thorac Oncol. 2018;13(5):636-648.

55. Mehta HJ, et al. Restoration of patency to central airways occluded by malignant endobronchial tumors using intratumoral injection of cisplatin. Ann Am Thorac Soc. 2015;12(9):1345-1350.

56. Mehta HJ, et al. Treatment of isolated mediastinal and hilar recurrence of lung cancer with bronchoscopic endobronchial ultrasound guided intratumoral injection of chemotherapy with cisplatin. Lung Cancer. 2015;90(3):542-547.

57. Jacks T, Fazeli A, Schmitt EM, Bronson RT, Goodell MA, Weinberg RA. Effects of an Rb mutation in the mouse. Nature. 1992;359(6393):295-300.

58. Safran M, Kim WY, Kung AL, Horner JW, DePinho RA, Kaelin WG. Mouse reporter strain for noninvasive bioluminescent imaging of cells that have undergone Cre-mediated recombination. $\mathrm{Mol}$ Imaging. 2003;2(4):297-302.

59. Meuwissen R, Linn SC, Linnoila RI, Zevenhoven J, Mooi WJ, Berns A. Induction of small cell lung cancer by somatic inactivation of both Trp53 and $\mathrm{Rb} 1$ in a conditional mouse model. Cancer Cell. 2003;4(3):181-189.

60. Dewals B, Myster F, Palmeira L, Gillet L, Ackermann M, Vanderplasschen A. Ex vivo bioluminescence detection of alcelaphine herpesvirus 1 infection during malignant catarrhal fever.
JVirol. 2011;85(14):6941-6954.

61. Ho VM, Schaffer BE, Karnezis AN, Park KS, Sage J. The retinoblastoma gene $\mathrm{Rb}$ and its family member $\mathrm{p} 130$ suppress lung adenocarcinoma induced by oncogenic K-Ras. Oncogene. 2009;28(10):1393-1399.

62. Ullman-Culleré MH, Foltz CJ. Body condition scoring: a rapid and accurate method for assessing health status in mice. Lab Anim Sci. 1999;49(3):319-323.

63. Phelps RM, et al. NCI-Navy Medical Oncology Branch cell line data base. JCell Biochem Suppl. 1996;24:32-91.

64. Bartee E, McFadden G. Human cancer cells have specifically lost the ability to induce the synergistic state caused by tumor necrosis factor plus interferon-beta. Cytokine. 2009;47(3):199-205.

65. Butler CR, et al. Vacuum-assisted decellularization: an accelerated protocol to generate tissue-engineered human tracheal scaffolds. Biomaterials. 2017;124:95-105.

66. Rakaee M, et al. Evaluation of tumor-infiltrating lymphocytes using routine $\mathrm{H} \& \mathrm{E}$ slides predicts patient survival in resected non-small cell lung cancer. Hum Pathol. 2018;79:188-198. 